\author{
Ecología
}

\title{
Análisis herpetofaunístico de la zona árida del estado de Hidalgo
}

\author{
Herpetofaunistic analysis of the arid zone of the Hidalgo State \\ Leonardo Fernández-Badillo ${ }^{\mathrm{a}, \mathrm{c}}$, Norma Leticia Manríquez-Morán ${ }^{\mathrm{a}}$, \\ Jesús Martín Castillo-Cerón ${ }^{\mathrm{b}}$ e Irene Goyenechea ${ }^{\mathrm{a}, \mathrm{b}, \mathrm{c}, *}$ \\ ${ }^{a}$ Laboratorio de Sistemática Molecular, Centro de Investigaciones Biológicas, Universidad Autónoma del Estado de Hidalgo, Ciudad del Conocimiento, \\ Km 4.5 carretera Pachuca-Tulancingo, Col. Carboneras, 42181 Mineral de la Reforma, Hidalgo, México \\ ${ }^{\mathrm{b}}$ Museo de Paleontología, Centro de Investigaciones Biológicas, Universidad Autónoma del Estado de Hidalgo, Ciudad del Conocimiento, \\ Km 4.5 carretera Pachuca-Tulancingo, Col. Carboneras, 42181 Mineral de la Reforma, Hidalgo, México \\ ${ }^{\mathrm{c}}$ Predio intensivo de manejo de vida silvestre X-plora reptilia, Reyna de la Esperanza 107, Col. Paseo de las Reinas, primera sección, \\ 42184, Mineral de la Reforma, Hidalgo, México
}

Recibido el 4 de mayo de 2015; aceptado el 21 de septiembre de 2015

Disponible en Internet el 24 de febrero de 2016

\begin{abstract}
Resumen
Se estudiaron la riqueza y la distribución de los anfibios y saurópsidos no aves de las 2 ecorregiones áridas de Hidalgo. Para ello, se realizó un listado taxonómico con información bibliográfica y de campo, se analizó la complementariedad entre ecorregiones y se comparó la composición de especies contra 5 zonas áridas de México. Además, se analizaron los atributos de historia natural de las especies, el estatus de conservación y el endemismo. Se registran 82 especies: 20 anfibios y 62 saurópsidos no aves. La ecorregión matorral central mexicano presentó la mayor riqueza con 65 especies: 29 exclusivas y 36 compartidas con la ecorregión matorral de la meseta central. Se registraron 62 especies en el matorral xerófilo, 2 en el bosque tropical caducifolio y 18 en ambos tipos de vegetación. La complementariedad entre ecorregiones fue de 55 para anfibios y 32.07 para saurópsidos no aves. La mayor similitud (52.4) se presentó con la zona árida de Querétaro. La mayoría de las especies son terrestres, insectívoras y ovíparas. Se registraron más anfibios nocturnos y más saurópsidos no aves diurnos. Finalmente, 18 especies se encuentran bajo protección especial, 16 amenazadas y 44 son endémicas de México.

Derechos Reservados (C) 2015 Universidad Nacional Autónoma de México, Instituto de Biología. Este es un artículo de acceso abierto distribuido bajo los términos de la Licencia Creative Commons CC BY-NC-ND 4.0.
\end{abstract}

Palabras clave: Anfibios; Saurópsidos no aves; Ecorregión árida; Listado taxonómico; Historia natural

\section{Abstract}

We studied richness and distribution of amphibians and non-avian sauropsids from the 2 arid ecoregions of Hidalgo. A taxonomic list with bibliographical and field information was used, complementarity among ecoregions was analyzed and the composition of species against 5 arid regions of Mexico was compared. In addition, we discussed natural history attributes of species, their conservation status, and endemism. We report 82 species (20 amphibian and 62 non-avian sauropsids). The eco-region Central Mexican Shrub showed the greatest richness (65 species; 29 unique and 36 shared with the Central Plateau Shrub eco-region). We found 62 species distributed only in xeric shrub, 2 in deciduous tropical forest and 18 in both types of vegetation. The complementarity between ecoregions was 55 for amphibians and 32.07 for non-avian sauropsids. The greater similarity (52.4) was found with the arid zone of Querétaro. Most species are terrestrial, insectivores, and oviparous. There were more nocturnal amphibians and more diurnal non-avian sauropsids registered. Finally, 18 species are under special protection, 16 threatened and 44 are endemic to Mexico.

All Rights Reserved (C) 2015 Universidad Nacional Autónoma de México, Instituto de Biología. This is an open access item distributed under the Creative Commons CC License BY-NC-ND 4.0.

Keywords: Amphibians; Non-avian sauropsids; Arid eco-region; Taxonomic list; Natural history

\footnotetext{
* Autor para correspondencia.

Correo electrónico: ireneg28@ gmail.com (I. Goyenechea).

La revisión por pares es responsabilidad de la Universidad Nacional Autónoma de México.
} 


\section{Introducción}

El estado de Hidalgo se ubica en la parte central de México $\left(19^{\circ} 35^{\prime} 52^{\prime \prime}\right.$ y $21^{\circ} 25^{\prime} 00^{\prime \prime} \mathrm{N}, 97^{\circ} 57^{\prime} 27^{\prime \prime}$ y $\left.99^{\circ} 51^{\prime} 51^{\prime \prime} \mathrm{O}\right)$, en el límite de las regiones biogeográficas Neártica y Neotropical, lo que, aunado a una accidentada y compleja topografía, da como resultado una gran variedad de ambientes, los cuales propician una excepcional biodiversidad. Estas características favorecen la presencia de 3 grupos principales de climas: $a$ ) el de los cálidos y semicálidos de la Huasteca hidalguense; $b$ ) los templados húmedos y subhúmedos de la sierra Madre Oriental (SMOr) y la Faja Volcánica Transmexicana (FVT), y $c$ ) el de los climas áridos y semiáridos de la porción hidalguense de la meseta central y la zona de la Barranca de Metztitlán (Martínez-Morales et al., 2007). Estos últimos son el tipo de clima predominante en Hidalgo, el cual se ha considerado uno de los estados más áridos del país (Ramírez-Bautista et al., 2014).

La zona árida de Hidalgo, que comprende el 39\% del estado, se encuentra integrada por 2 ecorregiones, la del matorral central mexicano (MCM) y la del matorral de la meseta central (MMC) de acuerdo con la propuesta de Olson et al. (2001). Los tipos de vegetación que se encuentran en las ecorregiones son: el matorral xerófilo (MX), predominante en el estado, con una superficie de 253,519 ha (12.7\%) y el bosque tropical caducifolio (BTC), con una superficie de 83,352 ha (4\%). Además, se considera que dentro de la zona árida de Hidalgo (ZAHGO) se cuenta con parches de bosque espinoso y de pastizal, que constituyen un porcentaje del 0.7 y el $0.1 \%$, respectivamente (Martínez-Morales et al., 2007).

Los estudios sobre la herpetofauna de Hidalgo han sido sesgados, ya que históricamente la zona árida ha sido menos estudiada que el resto de las regiones del estado. Sin embargo, se cuenta con los trabajos herpetofaunísticos llevados a cabo por Martín del Campo $(1936,1937)$ y varios estudios recientes (Campos-Rodríguez, Pérez-Valera y Flores-Leyva, 2010; Fernández-Badillo, 2008; Fernández-Badillo y Goyenechea, 2010; Gelover-Alfaro, Altamirano-Álvarez y Soriano, 2000; Gómez-Mendoza, 2007; Hernández-Pérez, 1997; HuitzilMendoza, 2007, 2010; Huitzil-Mendoza y Goyenechea, 2011; Lara-Parra, 2014; López-Mejía, 2011; Morales-Capellán, 2010; Roth-Monzón, 2010; Vite-Silva, 2008; Vite-Silva, RamírezBautista y Hernández-Salinas, 2010). Ninguno de los estudios mencionados comprende la totalidad de la región árida; el único trabajo que incluye una parte importante de esta zona es el de Camarillo-Rangel (1993). En este estudio el autor enlistó un total de 39 especies: 12 anfibios y 27 saurópsidos no aves, tradicionalmente conocidos como reptiles - tortugas, lagartijas y serpientes-. Por otra parte, Hernández-Salinas y RamírezBautista $(2012,2013)$ analizaron la distribución y la riqueza de los anfibios y de la herpetofauna de Hidalgo en 4 tipos de vegetación: bosque mesófilo de montaña, bosque de pino-encino, MX y bosque tropical perenifolio, y registraron los valores más bajos para el MX, con tan solo 6 especies de anfibios (Hernández-Salinas y Ramírez-Bautista, 2012) y 23 especies para la herpetofauna en total (Hernández-Salinas y RamírezBautista, 2013), a pesar de que el MX es el tipo de vegetación que presenta la mayor extensión dentro del estado. Por otro lado, Ramírez-Bautista et al. (2014) sugirieron la existencia de un total de 51 especies: 8 anfibios y 43 saurópsidos no aves, para los matorrales xerófilos de Hidalgo. Sin embargo, no tomaron en cuenta especies previamente registradas, por ejemplo, Fernández-Badillo y Goyenechea (2010).

Debido a que el conocimiento sobre la riqueza de los anfibios y saurópsidos no aves de la zona árida del estado de Hidalgo se encuentra disperso, los estudios recientes sobre la herpetofauna de la entidad no reúnen toda la información existente para esta zona, ya que se ha subestimado su riqueza; en el presente trabajo se presenta una actualización de la riqueza herpetofaunística de la ZAHGO y se analiza tanto la riqueza como la distribución de las especies por ecorregión: MCM y MMC, y en los 2 tipos de vegetación principales: MX y BTC, así como la complementariedad entre las ecorregiones y la similitud del área con 5 zonas áridas de México. Además, se analizaron algunos aspectos sobre la historia natural, el estado de conservación y el endemismo de estos grupos de vertebrados.

\section{Materiales y métodos}

La ZAHGO está integrada por 2 de las ecorregiones: el MCM y el MMC, propuestas por Olson et al. (2001). Los municipios del estado de Hidalgo fueron sobrepuestos en un mapa del estado, se recortó la capa municipal con la herramienta «cortar una capa a partir de otra» del programa ArcView 3.2 (ESRI, 1998) y se contabilizaron los municipios incluidos parcial o totalmente dentro de las ecorregiones. Con dicha información se generó un mapa de la ubicación de las ecorregiones áridas de Hidalgo, que comprende 60 de los 84 municipios (fig. 1).

Para elaborar la lista de especies se conjuntó información de trabajo de campo realizado entre 2006 y 2015, cuyos ejemplares recolectados se encuentran depositados en la Colección Herpetológica del Centro de Investigaciones Biológicas (CIB)-UAEH, así como de la revisión bibliográfica de los trabajos relacionados con la herpetofauna de la ZAHGO (Camarillo-Rangel, 1993; Campos-Rodríguez et al., 2010; Fernández-Badillo, 2008; Fernández-Badillo y Goyenechea, 2010; Gelover-Alfaro et al., 2000; Gómez-Mendoza, 2007; Hernández-Pérez, 1997; Huitzil-Mendoza, 2007, 2010; HuitzilMendoza y Goyenechea, 2011; López-Mejía, 2011; Lara-Parra, 2014; Marmolejo-Santillán, 1991; Mendoza-Quijano, 1990; Morales-Capellán, 2010; Pampa-Ramírez, 2010; Roth-Monzón, 2010; Vite-Silva, 2008; Vite-Silva et al., 2010), así como de aquellos trabajos que involucran a la herpetofauna del estado en general (Fernández-Badillo, Morales-Capellán y Goyenechea, 2011; Hernández-Salinas, 2009; Hernández-Salinas y RamírezBautista, 2012, 2013; Ramírez-Bautista et al., 2010, 2014), de los cuales se seleccionaron únicamente las especies registradas en la zona árida de Hidalgo. Se consultaron también notas científicas (Calzada-Arciniega y Hernández-Ríos, 2014; CamposRodríguez y Mendoza-Quijano, 2004; Goyenechea, MoralesCapellán y Fernández-Badillo, 2011a, b, c; Goyenechea, Vite y Mendiola-González, 2010; Hernández-Salinas, Cruz-Elizalde, Berriozábal-Islas y Goyenechea, 2011; Hernández-Salinas y Ramírez-Bautista, 2010; López-Mejía y Goyenechea, 2012a, b, c, d; Morales-Capellán, Fernández-Badillo y Goyenechea, 2011; 


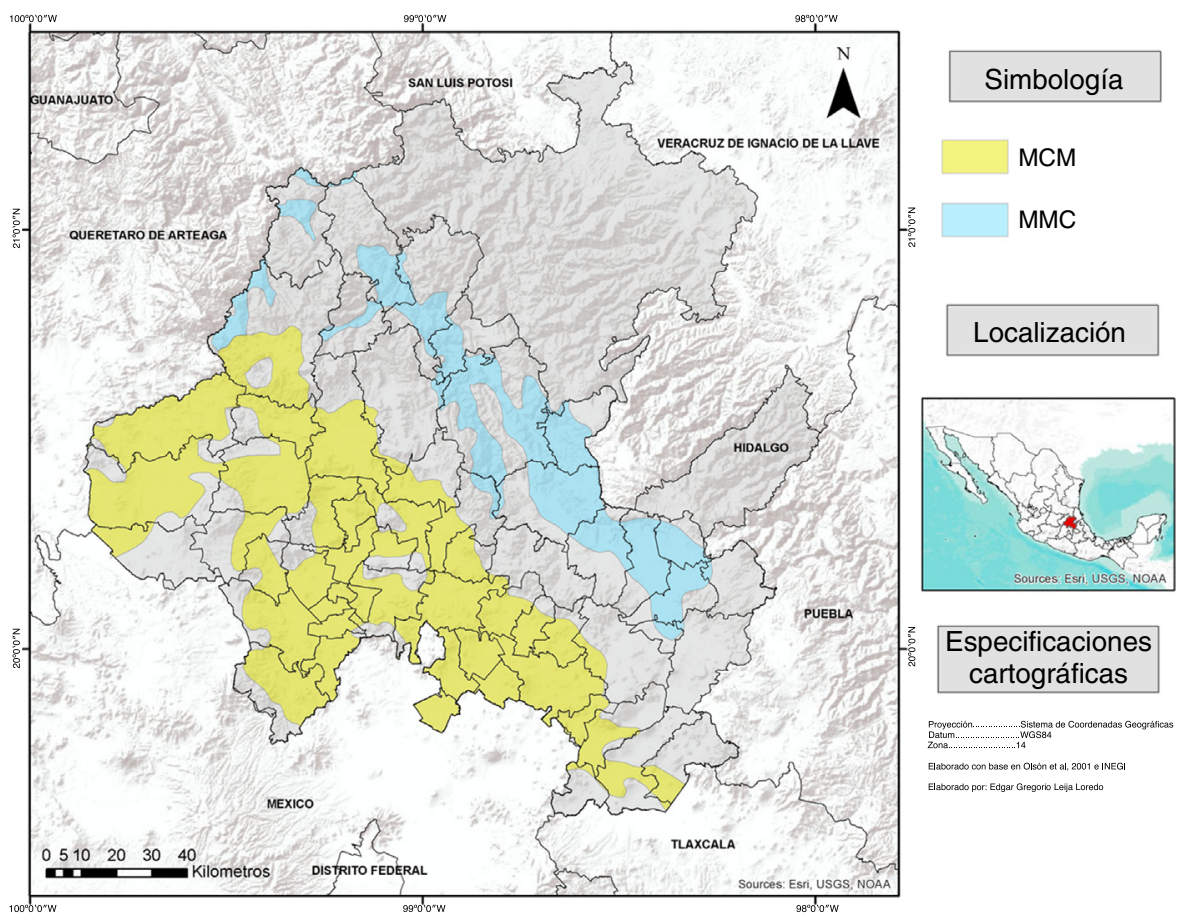

Figura 1. Mapa del estado de Hidalgo que muestra las 2 ecorregiones áridas. En el recuadro se observa la localización de Hidalgo en relación con el país. MCM: matorral central mexicano; MMC: matorral de la meseta central.

Roth-Monzón, Mendoza-Hernández y Flores-Villela, 2011), y se revisaron las bases de datos de las Colecciones de Anfibios y Reptiles del Centro de Investigaciones Biológicas (CIB) de la Universidad Autónoma del Estado de Hidalgo (HGOAN-152-0903, HGO-REP-153-0903), así como 1,468 registros de la base de datos generada en el proyecto «Diversidad biológica del estado de Hidalgo (tercera etapa)» Fomix-Conacyt Hidalgo 191908. La lista de especies obtenida fue depurada para eliminar registros dudosos y con errores de identificación taxonómica. Además, cuando fue posible, se revisaron los ejemplares recolectados para corroborar la identidad taxonómica. Los nombres de la especies se actualizaron con base en los trabajos de Flores-Villela y Canseco-Márquez (2004); Uetz (2015) y Wilson, Mata-Silva y Johnson (2013a, b).

Se analizó la distribución de las especies con matrices de presencia-ausencia de especies por ecorregión (MCM, MMC, MCM-MMC) y por tipo de vegetación (MX, BTC, BTC-MX), de acuerdo con la información del trabajo de campo, las bases de datos y la bibliografía. Se realizó un análisis de complementariedad de las 2 ecorregiones (MMC y MCM), el cual relaciona las especies del sitio A con las especies del sitio B y las especies compartidas entre ambos sitios (Colwell y Coddington, 1994).

Mediante un análisis de similitud se comparó la riqueza de especies de la ZAHGO contra 5 zonas áridas de México: el Valle de Tehuacán-Cuicatlán, Puebla (Canseco-Márquez y GutiérrezMayén, 2010); la porción árida de Tlaxcala (Fernández, Sánchez y Flores-Villela, 2006); Guadalcázar, San Luis Potosí (Hernández-Ibarra y Ramírez-Bautista, 2006); la zona árida de Querétaro (Nieto-Montes de Oca, 1999) y la zona árida de Aguascalientes (Vázquez-Díaz y Quintero-Díaz, 2005). Para este análisis se empleó el índice de Jaccard, ya que permite el uso de datos cualitativos como la presencia-ausencia de los organismos (Moreno, 2001) para establecer qué tan parecidos son los sitios en su composición de especies.

Adicionalmente, se obtuvieron 4 atributos de historia natural, con los cuales se clasificaron las especies en categorías, las cuales se establecieron sobre la base de la información derivada de las publicaciones de Dixon y Lemos-Espinal (2010); Fernández-Badillo et al. (2011); García-Vázquez et al. (2006); Lemos-Espinal y Dixon (2013); Lemos-Espinal y Smith (2008); Ramírez-Bautista, Hernández-Salinas, García-Vázquez, LeyteManrique y Canseco-Márquez (2009); Ramírez-Bautista et al. (2014) y Uribe-Peña, Ramírez-Bautista y Casas-Andreu (1999). Los datos se obtuvieron de la literatura anterior, así como de observaciones en campo: 1) hábitat, para el que se considera como terrestres a las especies que normalmente se encuentran sobre el suelo o enterradas, arborícolas para aquellas especies que suben a los árboles o a bardas de roca y acuáticas para aquellas que se localizan dentro o a las orillas de cuerpos de agua; 2) hábitos, categoría en la que se indica si la especie es diurna - que su actividad es durante el día—, nocturna — que está activa durante la noche-, diurna-crepuscular — está activa en el día y en el crepúsculo-, nocturna-crepuscular —activa durante el crepúsculo y la noche- o diurna-crepuscularnocturna - activa durante el día y la noche-; 3) tipo de alimentación, para el cual se considera insectívora - cuando se alimentan de invertebrados- o carnívora - cuando se alimentan de vertebrados-, y 4) tipo de paridad, dividido en ovíparas - cuando el embrión se desarrolla en el medio externo- y vivíparas - cuando el embrión se desarrolla dentro de la madre- La información anterior se obtuvo sobre la base de la revisión de diversos estudios herpetofaunísticos. 
Tabla 1

Composición herpetofaunística de la zona árida del estado de Hidalgo.

\begin{tabular}{lccc}
\hline Orden & Familia & Género & Especie \\
\hline Anura & 6 & 10 & 18 \\
Caudata & 2 & 2 & 2 \\
Total anfibios & 8 & 12 & 20 \\
Testudines & 1 & 1 & 2 \\
Squamata (sauria) & 6 & 9 & 19 \\
Squamata (serpentes) & 8 & 27 & 41 \\
Total saurópsidos no aves & 15 & 37 & 62 \\
\hline
\end{tabular}

El estado de conservación de las especies — amenazada, protegida, no considerada - así como el endemismo de las mismas —endémicas de México o no_- se determinaron basándose en la Nom-059-Semarnat-2010 (Semarnat, 2010) y Ramírez-Bautista et al. (2014), respectivamente.

\section{Resultados}

La zona árida del estado de Hidalgo cuenta con un total de 82 especies de anfibios y saurópsidos no aves (anexo). Los anfibios están representados por 2 órdenes, 8 familias, 12 géneros y 20 especies, de las cuales 18 son anuros y 2 corresponden a caudados. Los saurópsidos no aves se clasifican en 2 órdenes, 15 familias, 37 géneros y 62 especies, 41 de ellas son serpientes, 19 lagartijas y 2 tortugas (tabla 1 ).

Dentro de los anfibios, los anuros constituyen el orden con mayor riqueza. Las familias Bufonidae, Eleutherodactylidae, Hylidae y Ranidae cuentan cada una con 4 especies - $20 \%$ de la anfibiofauna registrada-, el resto de las familias, 2 de salamandras (Ambystomatidae y Plethodontidae) y 2 de anuros (Craugastoridae y Scaphiopodidae) presentan solo una especie (5\%). La mayor riqueza de saurópsidos no aves está representada por el orden Squamata, y dentro de él, las serpientes son el grupo más rico. La familia de serpientes con más especies es Colubridae con $18-29 \%$ del total de saurópsidos no aves registrados - , seguida por la familia de lagartijas Phrynosomatidae con 9 especies (15\%), le siguen las familias de serpientes Dipsadidae, Natricidae y Viperidae, con 6 especies cada una (9.7\%), la familia de lagartijas Anguidae, con 4 especies (6.5\%), una familia de tortugas (Kinosternidae) y 2 de lagartijas (Scincidae y Xantusidae), con 2 especies (3.2\%), y con 2 especies (1.6\%) 2 familias de lagartijas (Gekkonidae y Teiidae) y 4 de serpientes (Boidae, Elapidae, Leptotyphlopidae y Typhlopidae).

\section{Distribución por ecorregión}

El MCM de Hidalgo presenta una riqueza total de 65 especies: 18 anfibios y 47 saurópsidos no aves. Este total resulta de sumar 29 especies exclusivas y 36 compartidas con el MMC (tablas 2 y 3). Las especies que se distribuyen únicamente en esta ecorregión son 9 anfibios y 20 saurópsidos no aves. Los anfibios corresponden a 2 salamandras (Ambystoma velasci y Pseudoeurycea belli) y 7 anuros (Anaxyrus punctatus, Eleutherodactylus cystignatoides, Eleutherodactylus longipes, Eleutherodactylus nitidus, Lithobates catesbeianus, Lithobates

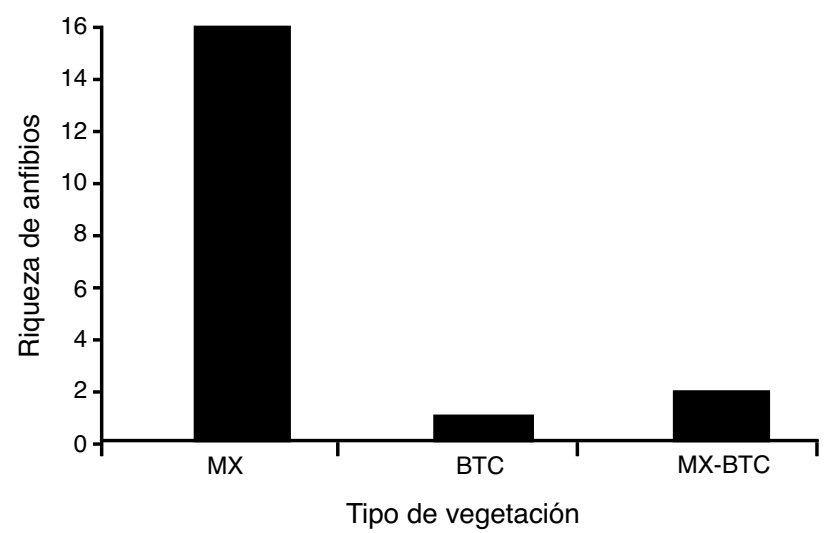

Figura 2. Riqueza de especies de anfibios registrados por tipo de vegetación. BTC: bosque tropical caducifolio; MX: matorral xerófilo; MX-BTC: matorral xerófilo y bosque tropical caducifolio.

montezumae y Smilisca baudini). Los saurópsidos no aves corresponden a una tortuga (Kinosternon hirtipes), 3 lagartijas (Barisia imbricata, Lepidophyma gaigeae y Sceloporus megalepidurus) y 16 serpientes (Conopsis nasus, Crotalus aquilus, Crotalus intermedius, Crotalus ravus, Crotalus scutulatus, Diadophis punctatus, Hypsiglena jani, Indotyphlops braminus, Lampropeltis mexicana, Lampropeltis ruthveni, Pantherophis emoryi, Salvadora bairdi, Salvadora grahamiae, Storeria hidalgoensis, Thamnophis melanogaster, Tropidodipsas sartorii).

Para los matorrales de la meseta central mexicana ubicados en Hidalgo, se registran 53 especies: 11 anfibios y 42 saurópsidos no aves. Este número se integra al sumar 36 compartidas con el MCM y 17 especies exclusivas, y (tablas 2 y 3), las cuales corresponden a 2 anuros (Craugastor augusti y Rhinella marina) y a 15 saurópsidos no aves: 5 lagartijas (Gerrhonotus liocephalus, Gerrhonotus ophiurus, Hemidactylus frenatus, Lepidophyma occulor y Scincella gemmingeri) y 10 serpientes (Boa constrictor, Geophis latifrontalis, Lampropeltis polyzona, Leptodeira maculata, Leptodeira septentrionalis, Nerodia rhombifer, Oxybelis aeneus, Rhadinaea gaigeae, Storeria dekayi y Thamnophis proximus).

\section{Distribución por tipo de vegetación}

Del total de las especies de anfibios registradas, $16-2$ salamandras y 14 anuros- se encuentran en los matorrales xerófilos $(80 \%)$, un anuro en el BTC (5\%) y 3 anuros en ambos tipos de vegetación (15\%; tabla 2; fig. 2). En el caso de los saurópsidos no aves, 44 especies - 2 tortugas, 13 lagartijas y 29 serpientes - se registran en los matorrales xerófilos (71\%), una - una lagartija - en el BTC (3.2\%) y 17 -5 lagartijas y 12 serpientes - en ambos tipos de vegetación (27\%; tabla 3; fig. 3).

\section{Complementariedad y similitud}

El análisis de complementariedad entre ambas ecorregiones para los anfibios mostró un valor de 55, mientras que para los saurópsidos no aves se obtuvo un valor de 32.07. Al comparar la riqueza herpetofaunística de la ZAHGO respecto a otras áreas 
Tabla 2

Lista de especies de anfibios y atributos de historia natural.

\begin{tabular}{|c|c|c|c|c|c|c|c|c|}
\hline Clasificación & Hábitat & Hábitos & T.A. & T.P. & End & NOM-059 & T.V. & Ecorregión \\
\hline \multicolumn{9}{|l|}{ Amphibia } \\
\hline \multicolumn{9}{|l|}{ Ambystomatidae } \\
\hline $\begin{array}{l}\text { Ambystoma velasci } \\
\text { Plethodontidae }\end{array}$ & TE-AC & DI-CR-NO & IN-CA & $\mathrm{O}$ & $\mathrm{E}$ & $\operatorname{Pr}$ & MX & $\mathrm{MCM}$ \\
\hline $\begin{array}{l}\text { Pseudoeurycea bellii } \\
\text { Bufonidae }\end{array}$ & $\mathrm{TE}$ & NO & IN & $\mathrm{O}$ & $\mathrm{E}$ & A & MX & $\mathrm{MCM}$ \\
\hline Anaxyrus punctatus & TE & NO & IN & $\mathrm{O}$ & $\mathrm{NE}$ & $\mathrm{Nc}$ & MX & MCM \\
\hline Incilius occidentalis & TE-AC & NO & IN & $\mathrm{O}$ & $\mathrm{E}$ & $\mathrm{Nc}$ & MX & MCM-MMC \\
\hline Incilius valliceps & TE-AC & NO & IN & $\mathrm{O}$ & $\mathrm{NE}$ & $\mathrm{Nc}$ & MX-BTC & MCM-MMC \\
\hline $\begin{array}{l}\text { Rhinella marina } \\
\text { Craugastoridae }\end{array}$ & $\mathrm{TE}$ & NO & IN-CA & $\mathrm{O}$ & $\mathrm{NE}$ & $\mathrm{Nc}$ & BTC & MMC \\
\hline $\begin{array}{l}\text { Craugastor augusti } \\
\text { Eleutherodactylidae }\end{array}$ & $\mathrm{TE}$ & NO & IN & $\mathrm{O}$ & $\mathrm{NE}$ & $\mathrm{Nc}$ & MX & MMC \\
\hline Eleutherodactylus cystignatoides & $\mathrm{TE}$ & NO & IN & $\mathrm{O}$ & $\mathrm{NE}$ & $\mathrm{Nc}$ & MX & MCM \\
\hline Eleutherodactylus longipes & $\mathrm{TE}$ & NO & IN & $\mathrm{O}$ & $\mathrm{E}$ & $\mathrm{Nc}$ & MX & $\mathrm{MCM}$ \\
\hline Eleutherodactylus nitidus & AR & NO & IN & $\mathrm{O}$ & $\mathrm{E}$ & $\mathrm{Nc}$ & MX & $\mathrm{MCM}$ \\
\hline $\begin{array}{l}\text { Eleutherodactylus verrucipes } \\
\text { Hylidae }\end{array}$ & \multicolumn{7}{|c|}{ Hylidae } & MCM-MMC \\
\hline Ecnomiohyla miotympanum & $\mathrm{AR}$ & NO & IN & $\mathrm{O}$ & E & $\mathrm{Nc}$ & MX-BTC & MCM-MMC \\
\hline Hyla arenicolor & $\mathrm{TE}$ & DI-CR-NO & IN & $\mathrm{O}$ & $\mathrm{NE}$ & $\mathrm{Nc}$ & MX & MCM-MMC \\
\hline Hyla eximia & TE & DI-CR & IN & $\mathrm{O}$ & $\mathrm{E}$ & $\mathrm{Nc}$ & MX & MCM-MMC \\
\hline $\begin{array}{l}\text { Smilisca baudini } \\
\text { Ranidae }\end{array}$ & $\mathrm{AR}$ & NO & IN & $\mathrm{O}$ & $\mathrm{NE}$ & $\mathrm{Nc}$ & MX & MCM \\
\hline Lithobates berlandieri & TE-AC & NO & IN & $\mathrm{O}$ & $\mathrm{NE}$ & $\operatorname{Pr}$ & MX-BTC & MCM-MMC \\
\hline Lithobates catesbeianus & TE-AC & NO & IN-CA & $\mathrm{O}$ & $\mathrm{NE}$ & $\mathrm{Nc}$ & MX & $\mathrm{MCM}$ \\
\hline Lithobates montezumae & TE-AC & NO & IN & $\mathrm{O}$ & $\mathrm{E}$ & $\operatorname{Pr}$ & MX & MCM \\
\hline $\begin{array}{l}\text { Lithobates spectabilis } \\
\text { Scaphiopodidae }\end{array}$ & TE-AC & NO & IN & $\mathrm{O}$ & $\mathrm{E}$ & $\mathrm{Nc}$ & MX & MCM-MMC \\
\hline Spea multiplicata & TE & NO & IN & $\mathrm{O}$ & $\mathrm{NE}$ & $\mathrm{Nc}$ & MX & MCM-MMC \\
\hline
\end{tabular}

A: amenazado; AC: acuático; AR: arborícola; BTC: bosque tropical caducifolio; CA: carnívoro; CR: crepuscular; DI: diurno; E: endémico; End: endemismos; IN: insectívoro; Nc: no considerado; NE: no endémico; NO: nocturno; MCM: matorral central mexicano; MMC: matorral de la meseta central; MX: matorral xerófilo; O: ovíparo; Pr: protección especial; T.A.: tipo de alimentación; TE: terrestre; T.P.: tipo de paridad; T.V.: tipo de vegetación; VI: vivíparo.

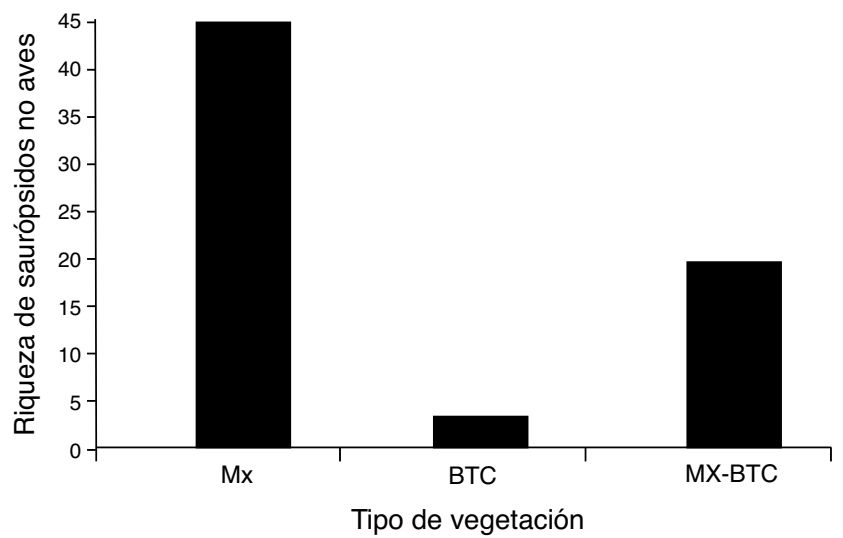

Figura 3. Riqueza de especies de saurópsidos no aves registradas por tipo de vegetación.

BTC: bosque tropical caducifolio; MX: matorral xerófilo; MX-BTC: matorral xerófilo y bosque tropical caducifolio.

del país (fig. 4), el valor de similitud más alto se obtuvo entre la ZAHGO y la zona árida de Querétaro (52.9\%). A continuación, con la región árida de Aguascalientes (29.5\%), seguida de la comparación con el Valle de Tehuacán-Cuicatlán (26.4\%), después con San Luis Potosí (26.5\%) y, finalmente con la porción xerófila de Tlaxcala $(22.2 \%)$

\section{Atributos de historia natural}

En los distintos atributos de historia natural analizados para los anfibios (fig. 5 a; tabla 2) se encontró que del total de las especies, 10 - una salamandra y 9 anuros - son terrestres (50\%), 7 - una salamandra y 6 anuros—-son terrestres-acuáticas (35\%) y 3 anuros son arborícolas (15\%). Respecto a los hábitos (fig. 5 b), 17 especies de anuros son nocturnas (85\%), 2 - una salamandra y un anuro- son diurnas-crepuscularesnocturnas $(10 \%)$ y una especie de anuro es diurna-crepuscular (5\%). Se registran 17 anuros insectívoros (85\%) y 3 especies -una salamandra y 2 anuros - insectívoras-carnívoras (15\%; fig. 5 c). El tipo de paridad encontrado para las 20 especies es ovíparo.

Los atributos de historia natural de los saurópsidos no aves muestran que (fig. 6; tabla 3) para el caso del hábitat (fig. 6 a), 39 especies - 9 lagartijas y 30 serpientes - son terrestres (63\%), 14 -9 lagartijas y 5 serpientes - son terrestres-arborícolas (23\%), $7-2$ tortugas y 5 serpientes - son terrestres-acuáticas (11\%) 
Tabla 3

Lista de especies de saurópsidos no aves y atributos de historia natural.

\begin{tabular}{|c|c|c|c|c|c|c|c|c|}
\hline Clasificación & Hábitat & Hábitos & T.A. & T.P. & End & NOM-059 & T.V. & Ecorregión \\
\hline $\begin{array}{l}\text { Reptilia } \\
\text { Testudine } \\
\text { Kinosternidae }\end{array}$ & & & & & & & & \\
\hline Kinosternon hirtipes & TE-AC & DI & IN & $\mathrm{O}$ & $\mathrm{NE}$ & $\mathrm{Nc}$ & MX & $\mathrm{MCM}$ \\
\hline $\begin{array}{l}\text { Kinosternon integrum } \\
\text { Sauria } \\
\text { Anguidae }\end{array}$ & TE-AC & DI & IN-CA & $\mathrm{O}$ & $\mathrm{E}$ & $\operatorname{Pr}$ & MX & MCM-MMC \\
\hline Barisia imbricata & $\mathrm{TE}$ & DI & IN & V & $\mathrm{E}$ & $\operatorname{Pr}$ & MX & MCM \\
\hline Gerrhonotus infernalis & TE-AR & DI-CR & IN-CA & $\mathrm{O}$ & $\mathrm{E}$ & $\mathrm{Nc}$ & MX & MCM-MMC \\
\hline Gerrhonotus liocephalus & TE-AR & DI-CR-NO & IN & $\mathrm{O}$ & $\mathrm{E}$ & $\mathrm{Nc}$ & MX-BTC & MMC \\
\hline $\begin{array}{l}\text { Gerrhonotus ophiurus } \\
\text { Gekkonidae }\end{array}$ & TE-AR & DI-CR & IN & $\mathrm{O}$ & $\mathrm{E}$ & $\mathrm{Nc}$ & $\mathrm{MX}$ & MMC \\
\hline $\begin{array}{l}\text { Hemidactylus frenatus } \\
\text { Phrynosomatidae }\end{array}$ & $\mathrm{AR}$ & NO & IN & $\mathrm{O}$ & $\mathrm{NE}$ & $\mathrm{Nc}$ & BTC & MMC \\
\hline Phrynosoma orbiculare & $\mathrm{TE}$ & DI & IN & $\mathrm{O}$ & $\mathrm{E}$ & A & MX & MCM-MMC \\
\hline Sceloporus grammicus & TE-AR & DI & IN & $\mathrm{V}$ & $\mathrm{E}$ & $\operatorname{Pr}$ & MX & MCM-MMC \\
\hline Sceloporus megalepidurus & TE-AR & DI & IN & $\mathrm{V}$ & $\mathrm{E}$ & $\operatorname{Pr}$ & MX & $\mathrm{MCM}$ \\
\hline Sceloporus minor & TE-AR & DI & IN & $\mathrm{V}$ & $\mathrm{NE}$ & $\mathrm{Nc}$ & MX-BTC & MCM-MMC \\
\hline Sceloporus тисronatus & TE-AR & DI & IN & $\mathrm{V}$ & E & $\mathrm{Nc}$ & $\mathrm{MX}$ & MCM-MMC \\
\hline Sceloporus parvus & $\mathrm{TE}$ & DI & IN & $\mathrm{O}$ & $\mathrm{E}$ & $\mathrm{Nc}$ & MX & MCM-MMC \\
\hline Sceloporus spinosus & TE-AR & DI & IN & $\mathrm{O}$ & $\mathrm{E}$ & $\mathrm{Nc}$ & MX & MCM-MMC \\
\hline Sceloporus torquatus & TE-AR & DI & IN & $\mathrm{V}$ & $\mathrm{E}$ & $\mathrm{Nc}$ & MX & MCM-MMC \\
\hline $\begin{array}{l}\text { Sceloporus variabilis } \\
\text { Scincidae }\end{array}$ & $\mathrm{TE}$ & DI & IN & $\mathrm{O}$ & $\mathrm{NE}$ & $\mathrm{Nc}$ & MX-BTC & MCM-MMC \\
\hline Scincella gemmingeri & $\mathrm{TE}$ & DI & IN & $\mathrm{O}$ & $\mathrm{E}$ & $\operatorname{Pr}$ & MX-BTC & MMC \\
\hline $\begin{array}{l}\text { Plestiodon lynxe } \\
\text { Teiidae }\end{array}$ & $\mathrm{TE}$ & DI & IN & $\mathrm{V}$ & $\mathrm{E}$ & $\mathrm{Nc}$ & $\mathrm{MX}$ & MCM-MMC \\
\hline $\begin{array}{l}\text { Aspidoscelis gularis } \\
\text { Xantusidae }\end{array}$ & $\mathrm{TE}$ & DI & IN & $\mathrm{O}$ & $\mathrm{NE}$ & $\mathrm{Nc}$ & MX-BTC & MCM-MMC \\
\hline Lepidophyma gaigeae & $\mathrm{TE}$ & NO & IN & $\mathrm{V}$ & $\mathrm{E}$ & $\operatorname{Pr}$ & MX & $\mathrm{MCM}$ \\
\hline $\begin{array}{l}\text { Lepidophyma occulor } \\
\text { Serpentes }\end{array}$ & TE & NO & IN & $\mathrm{V}$ & $\mathrm{E}$ & $\operatorname{Pr}$ & MX & MMC \\
\hline Boidae & & & & & & & & \\
\hline $\begin{array}{l}\text { Boa constrictor } \\
\text { Colubridae }\end{array}$ & TE-AR & DI-CR-NO & $\mathrm{CA}$ & $\mathrm{V}$ & NE & A & MX-BTC & MMC \\
\hline Conopsis lineata & $\mathrm{TE}$ & DI & IN & $\mathrm{V}$ & $\mathrm{E}$ & A & MX & MCM-MMC \\
\hline Conopsis nasus & $\mathrm{TE}$ & DI & IN & $\mathrm{V}$ & $\mathrm{E}$ & $\mathrm{Nc}$ & $\mathrm{MX}$ & MCM \\
\hline Drymarchon melanurus & TE & DI & $\mathrm{CA}$ & $\mathrm{O}$ & $\mathrm{NE}$ & $\mathrm{Nc}$ & MX-BTC & MCM-MMC \\
\hline Ficimia hardyi & TE & DI & IN & $\mathrm{O}$ & $\mathrm{E}$ & $\mathrm{Nc}$ & MX & MCM-MMC \\
\hline Lampropeltis mexicana & TE & NO-CR & $\mathrm{CA}$ & $\mathrm{O}$ & $\mathrm{E}$ & A & MX & $\mathrm{MCM}$ \\
\hline Lampropeltis polyzona & $\mathrm{TE}$ & NO-CR & $\mathrm{CA}$ & $\mathrm{O}$ & $\mathrm{NE}$ & A & MX & MMC \\
\hline Lampropeltis ruthveni & $\mathrm{TE}$ & NO-CR & $\mathrm{CA}$ & $\mathrm{O}$ & $\mathrm{E}$ & A & MX & $\mathrm{MCM}$ \\
\hline Masticophis schotti & TE-AR & DI & $\mathrm{CA}$ & $\mathrm{O}$ & $\mathrm{NE}$ & $\mathrm{Nc}$ & MX & MCM-MMC \\
\hline Oxybelis aeneus & AR & DI & $\mathrm{CA}$ & $\mathrm{O}$ & $\mathrm{NE}$ & $\mathrm{Nc}$ & MX-BTC & MMC \\
\hline Pantherophis emoryi & TE & DI & $\mathrm{CA}$ & $\mathrm{O}$ & $\mathrm{NE}$ & $\mathrm{Nc}$ & $\mathrm{MX}$ & $\mathrm{MCM}$ \\
\hline Pituophis deppei & TE & DI & $\mathrm{CA}$ & $\mathrm{O}$ & $\mathrm{E}$ & A & MX & MCM-MMC \\
\hline Rhadinaea gaigeae & $\mathrm{TE}$ & DI & IN & $\mathrm{O}$ & $\mathrm{E}$ & $\mathrm{Nc}$ & MX & MMC \\
\hline Salvadora bairdi & $\mathrm{TE}$ & DI & $\mathrm{CA}$ & $\mathrm{O}$ & $\mathrm{E}$ & $\operatorname{Pr}$ & MX & $\mathrm{MCM}$ \\
\hline Salvadora grahamiae & $\mathrm{TE}$ & DI & $\mathrm{CA}$ & $\mathrm{O}$ & $\mathrm{NE}$ & $\mathrm{Nc}$ & MX & $\mathrm{MCM}$ \\
\hline Senticolis triaspis & TE-AR & DI & $\mathrm{CA}$ & $\mathrm{O}$ & $\mathrm{NE}$ & $\mathrm{Nc}$ & MX-BTC & MCM-MMC \\
\hline Tantilla bocourti & TE & NO & IN & $\mathrm{O}$ & $\mathrm{E}$ & $\mathrm{Nc}$ & MX-BTC & MCM-MMC \\
\hline Trimorphodon tau & TE-AR & NO-CR & $\mathrm{CA}$ & $\mathrm{O}$ & $\mathrm{E}$ & $\mathrm{Nc}$ & $\mathrm{MX}$ & MCM-MMC \\
\hline $\begin{array}{l}\text { Tropidodipsas sartorii } \\
\text { Dipsadidae }\end{array}$ & $\mathrm{TE}$ & NO-CR & IN & $\mathrm{O}$ & $\mathrm{NE}$ & $\operatorname{Pr}$ & MX & $\mathrm{MCM}$ \\
\hline Diadophis punctatus & TE & NO-CR & IN-CA & $\mathrm{O}$ & $\mathrm{NE}$ & $\mathrm{Nc}$ & MX & $\mathrm{MCM}$ \\
\hline Geophis latifrontalis & $\mathrm{TE}$ & DI & IN & $\mathrm{O}$ & $\mathrm{E}$ & $\mathrm{Nc}$ & MX-BTC & MMC \\
\hline Hypsiglena jani & $\mathrm{TE}$ & NO & $\mathrm{CA}$ & $\mathrm{O}$ & $\mathrm{NE}$ & $\mathrm{Nc}$ & $\mathrm{MX}$ & $\mathrm{MCM}$ \\
\hline Leptodeira maculata & $\mathrm{TE}$ & NO & $\mathrm{CA}$ & $\mathrm{O}$ & $\mathrm{E}$ & $\mathrm{Nc}$ & MX & MMC \\
\hline Leptodeira septentrionalis & $\mathrm{TE}$ & NO & $\mathrm{CA}$ & $\mathrm{O}$ & $\mathrm{NE}$ & $\mathrm{Nc}$ & MX & MMC \\
\hline $\begin{array}{l}\text { Leptophis mexicanus } \\
\text { Elapidae }\end{array}$ & TE-AR & DI & $\mathrm{CA}$ & $\mathrm{O}$ & $\mathrm{NE}$ & A & MX & MCM-MMC \\
\hline Micrurus tener & $\mathrm{TE}$ & NO-CR & $\mathrm{CA}$ & $\mathrm{O}$ & $\mathrm{NE}$ & $\operatorname{Pr}$ & MX-BTC & MCM-MMC \\
\hline
\end{tabular}


Tabla 3 (Continuación)

\begin{tabular}{|c|c|c|c|c|c|c|c|c|}
\hline Clasificación & Hábitat & Hábitos & T.A. & T.P. & End & NOM-059 & T.V. & Ecorregión \\
\hline $\begin{array}{l}\text { Rena dulcis } \\
\text { Natricidae }\end{array}$ & $\mathrm{TE}$ & NO & IN & $\mathrm{O}$ & NE & $\mathrm{Nc}$ & MX-BTC & MCM-MMC \\
\hline Storeria dekayi & TE & DI-CR & IN & $\mathrm{V}$ & NE & $\mathrm{Nc}$ & MX & MMC \\
\hline Storeria hidalgoensis & $\mathrm{TE}$ & DI-CR-NO & IN & $\mathrm{V}$ & E & $\mathrm{Nc}$ & MX & MCM \\
\hline Thamnophis cyrtopsis & TE-AC & DI & CA & V & $\mathrm{NE}$ & A & MX-BTC & MCM-MMC \\
\hline $\begin{array}{l}\text { Thamnophis proximus } \\
\text { Typhlopidae }\end{array}$ & TE-AC & DI & $\mathrm{CA}$ & $\mathrm{V}$ & NE & A & MX-BTC & MMC \\
\hline $\begin{array}{l}\text { Indotyphlops braminus } \\
\text { Viperidae }\end{array}$ & $\mathrm{TE}$ & NO & IN & $\mathrm{O}$ & $\mathrm{NE}$ & $\mathrm{Nc}$ & MX & $\mathrm{MCM}$ \\
\hline Crotalus aquilus & $\mathrm{TE}$ & DI-CR & CA & $\mathrm{V}$ & $\mathrm{E}$ & $\operatorname{Pr}$ & $\mathrm{MX}$ & MCM \\
\hline Crotalus atrox & $\mathrm{TE}$ & DI-CR-NO & CA & $\mathrm{V}$ & NE & $\operatorname{Pr}$ & MX-BTC & MCM-MMC \\
\hline
\end{tabular}

A: amenazado; AC: acuático; AR: arborícola; BTC: bosque tropical caducifolio; CA: carnívoro; DI: diurno; DI-CR: diurno crepuscular; DI-CR-NO: diurno crepuscular nocturno; E: endémico; End: endemismos; IN: insectívoro; Nc: no considerado; NE: no endémico; NO: nocturno; NO-CR: nocturno crepuscular; MCM: matorral central mexicano; MMC: matorral de la meseta central; MX: matorral xerófilo; O: ovíparo; Pr: protección especial; T.A.: tipo de alimentación; TE: terrestre; T.P.: tipo de paridad; End: endemismos; T.V.: tipo de vegetación; VI: vivíparo.

y 2 especies - una lagartija y una serpiente- son arborícolas (3.2\%). En cuanto a los hábitos (fig. 6 b), 35 especies -2 tortugas, 13 lagartijas y 20 serpientes - son diurnas (56\%), 9 -3 lagartijas y 6 serpientes - son nocturnas $(15 \%)$, 7 serpientes son nocturnas-crepusculares (11\%), 7 especies —una lagartija y 6 serpientes - son diurnas-crepusculares-nocturnas (11\%) y 4 -2 lagartijas y 2 serpientes - son de hábitos diurnoscrepusculares $(6.5 \%)$. Con respecto a los hábitos alimentarios (fig. 6 c), se registraron 30 taxones - una tortuga, 18 lagartijas y 11 serpientes- insectívoros (48\%), 29 serpientes carnívoras $(47 \%)$ y 3 especies - una tortuga, una lagartija y una serpiente- insectívoras-carnívoras (4.8\%). En cuanto al tipo de paridad (fig. 6 d), 37 especies - 2 tortugas, 10 lagartijas y 25 serpientes - son ovíparas (60\%) y 25 especies - 9 lagartijas y 16 serpientes - son vivíparas (40\%).

\section{Estado de conservación y endemismo}

Respecto al estado de conservación de las especies de anfibios (fig. $5 \mathrm{~d}$; tabla 2), únicamente 4 especies —una salamandra, $A$. velasci, y 3 anuros, Eleutherodactylus verrucipes, Lithobates berlandieri y L. montezumae - se encuentran bajo la categoría de protección especial (21\%) y solo una especie de salamandra se registra como amenazada (Pseudoeurycea belli; 5.3\%). De las 15 especies de anuros restantes $(75 \%)$ no hay datos sobre su estado de conservación. En relación con la cantidad de especies endémicas, 10 de las 20 especies registradas en la ZAHGO -2 salamandras y 8 anuros- son endémicas de México, es decir, el 29.4\% de los anfibios endémicos para México están registrados en Hidalgo.

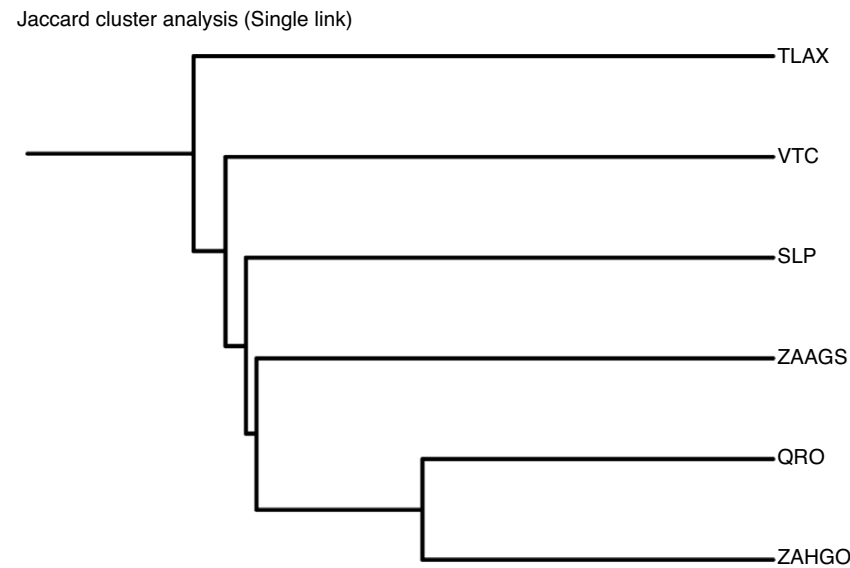

$\begin{array}{lll}0 . \% \text { Similitud } & 50 . & 100\end{array}$

Figura 4. Análisis de similitud de la herpetofauna de la zona árida de Hidalgo y otras zonas áridas de México.

QRO: Querétaro; SLP: Guadalcázar, San Luis Potosí; TLAX: Tlaxcala; VTC: Valle de Tehuacán-Cuicatlán; ZAAGS: zona árida de Aguascalientes; ZAHGO: zona árida de Hidalgo.

El estado de conservación de las especies de saurópsidos no aves (fig. 6 e; tabla 3) se presenta de la siguiente manera: 14 especies - una tortuga, 6 lagartijas y 7 serpientes - se encuentran bajo protección especial (23\%), 14 -una lagartija y 13 serpientes - bajo la categoría de amenazada (23\%) y las 34 especies restantes - una tortuga, 12 lagartijas y 21 serpientesno se encuentran enlistadas bajo alguna categoría (55\%). Por otro lado, 33 de las 62 especies son endémicas de México —una 

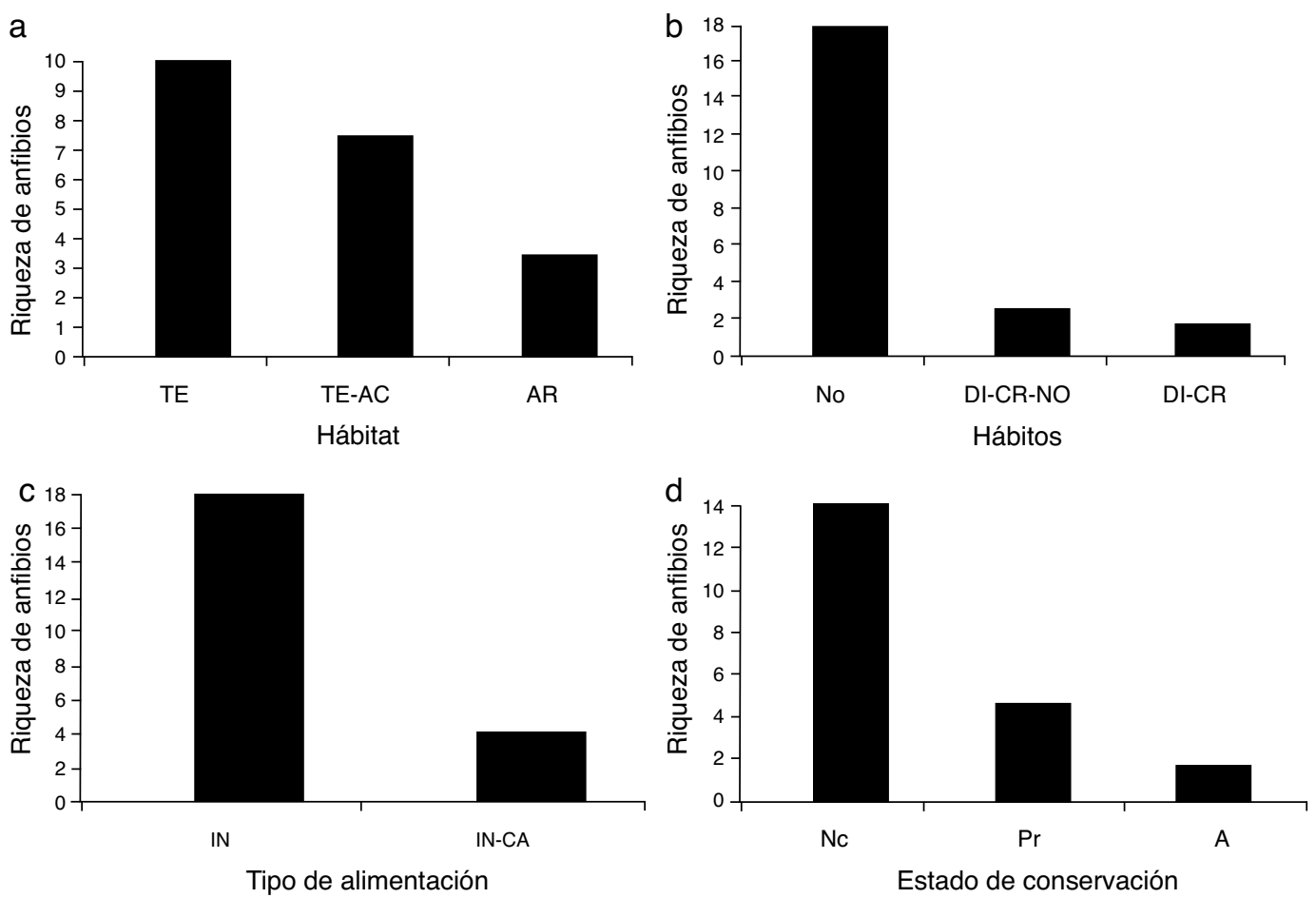

Figura 5. Riqueza de anfibios por atributo de historia natural. a) Hábitat; b) hábitos; c) tipo de alimentación; d) estado de conservación.

tortuga, 15 lagartijas y 17 serpientes-, es decir, que el $54 \%$ de los saurópsidos no aves endémicas de México habitan en Hidalgo.

\section{Discusión}

La riqueza herpetofaunística ( 82 especies) para la ZAHGO incluye 44 especies adicionales a la lista publicada por Camarillo-Rangel (1993), lo que demuestra lo desatendida que se encontraba esta región. La riqueza herpetofaunística en la ZAHGO puede explicarse por la conjunción de varios factores y evidencias biogeográficas. Primero, la ZAHGO se encuentra inmersa dentro de las provincias del Altiplano Mexicano, Eje Volcánico Transmexicano y SMOr (Morrone, 2005), y se ha considerado que pertenece al extremo sur del desierto Chihuahuense junto con las zonas áridas de Querétaro (Miranda y Hernández, 1963), denominada zona árida queretana-hidalguense (Hernández, 2006; Granados-Sánchez, Sánchez-Gonzáles, Granados-Victorino y Borja-de la Rosa, 2011), por lo que es posible observar algunas especies ampliamente distribuidas en el desierto Chihuahuense (C. scutulatus; Campbell y Lamar, 2004), así como especies de las zonas áridas de Norteamérica (A. punctatus, Crotalus atrox; Bryson, Jaeger, Lemos-Espinal y Lazcano, 2012; Campbell y Lamar, 2004), que se ubican también en la ZAHGO. Segundo, existe un componente tropical que conforma la herpetofauna de la ZAHGO, identificado previamente por Camarillo-Rangel $(1993 ; 2002)$ y Campos-Rodríguez et al. (2010), por lo que es posible encontrar especies ampliamente distribuidas en los trópicos mexicanos $(R$. marina, Drymarchon melanurus, $H$. frenatus, Nerodia rhombifer, O. aeneus, Sceloporus variabilis, T. proximus), algunas de las cuales se consideran características de la provincia del Golfo de México (Sceloporus variabilis; Espinosa-Organista y Ocegueda, 2008). Este componente tropical se ve mejor representado en la porción hidalguense del MMC, debido a que algunas especies se distribuyen desde la Planicie Costera del Golfo hasta la Meseta Central a través de las barrancas formadas por los ríos Moctezuma, Amajac y Metztitlán (Camarillo-Rangel, 1993; Campos-Rodríguez et al., 2010). Tercero, es posible encontrar especies características de ambientes templados, propias de la Zona de Transición Mexicana (que incluye las provincias de la sierra Madre Occidental [SMOcc], SMOr y FVT), por ejemplo, Thamnophis eques y T. melanogaster de la SMOcc. Lepidophyma gaigeae, L. occulor, Storeria hidalgoensis de la SMOr. Eleutherodactylus verrucipes, Ficimia hardyi, G. latifrontalis y R. gaigeae (Canseco-Márquez, Mendoza-Quijano y GutiérrezMayén, 2004) de la SMOr. L. montezumae (Espinosa-Organista y Ocegueda, 2008; Ramírez-Bautista et al., 2009), A. velasci, Lithobates spectabilis y Sceloporus mucronatus (Ramírez-Bautista et al., 2009) de la FVT.

En cuanto a los tipos de vegetación de la ZAHGO, se observó un mayor número de especies en los matorrales xerófilos (80) respecto a los bosques tropicales caducifolios (20), contrario a lo que documentan Vite-Silva et al. (2010), quienes en su estudio de la Reserva de la Biosfera de Meztitlán señalan el BTC como el tipo de vegetación con mayor número de especies (14), mientras que en el MX solo encontraron 9. Las diferencias entre ambos estudios pueden deberse a la falta de muestreos en el BTC de las ZAHGO, por lo que es posible que conforme se incrementen los estudios en ese tipo de vegetación, se registre una mayor riqueza de especies. Por otro lado, Ramírez-Bautista et al. (2014) registran 51 especies en MX y 46 especies en BTC. 

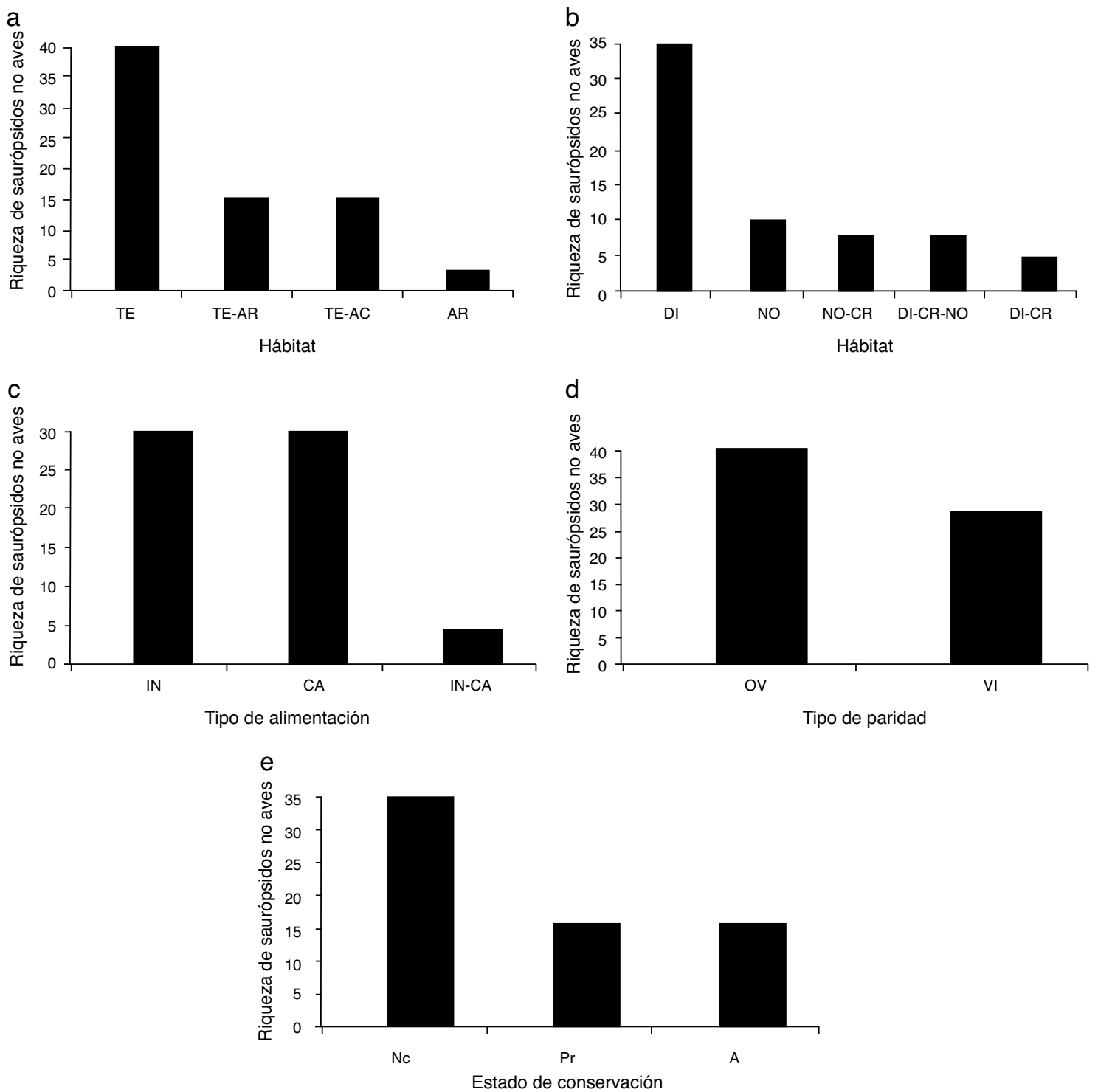

Figura 6. Riqueza de especies de saurópsidos no aves por atributo de historia natural. a) Hábitat; b) hábitos; c) tipo de alimentación; d) modo reproductor; e) estado de conservación.

La mayor riqueza de especies en el BTC registrada en el trabajo citado comparada con los resultados obtenidos aquí (46 vs. 20) puede deberse a que varios registros de los autores previos provienen de municipios como Molango, Yahualica, Atlapexco, Xochiatipán, Pisaflores y Chapulhuacán, que quedan fuera de la ZAHGO, de acuerdo con el método descrito en este trabajo para la delimitación de la zona.

El análisis de complementariedad realizado mostró que ambas ecorregiones difieren en más del $40 \%$ de sus especies tanto para anfibios como para saurópsidos no aves, de tal manera que cada ecorregión alberga especies exclusivas que las diferencian claramente. La relación existente entre la herpetofauna de la zona árida y las otras 5 áreas áridas seleccionadas muestra distintos valores de similitud. La mayor similitud se encontró entre la zona de estudio (ZAHGO) y la zona árida de Querétaro (52.4\%), lo cual ya había sido detectado por Camarillo-Rangel (1993) y Huitzil-Mendoza (2010). Esto puede explicarse por la colindancia de estas zonas, por la aparente uniformidad en los ambientes que poseen (Huitzil-Mendoza, 2010) y porque ambas zonas forman parte de la misma ecorregión (MCM), y, en conjunto, han sido consideradas como una subdivisión del desierto Chihuahuense (Hernández, 2006; Granados-Sánchez et al., 2011). Por otro lado, se confirmó la escasa similitud entre la ZAHGO con el Valle de Tehuacán-Cuicatlán y con la porción árida de Tlaxcala (Camarillo-Rangel, 1993, Fernández-Badillo, 2008; Fernández-Badillo y Goyenechea, 2010 y Huitzil-Mendoza, 2010). Además, no se observaron similitudes importantes con San Luis Potosí y la zona árida de Aguascalientes, como lo habían mencionado Camarillo-Rangel (1993) y Huitzil-Mendoza (2010). Aunque cierto porcentaje de la herpetofauna de la ZAHGO se comparte con otras regiones áridas cercanas, esta zona muestra una composición de especies particular, por lo que es necesario continuar con su estudio para tener un mejor entendimiento de la distribución 
de las especies y desarrollar estrategias para su conservación.

En cuanto al hábitat que utilizan las distintas especies en la zona árida de Hidalgo, se encontró que la mayoría son terrestres. Esto puede deberse a la ausencia de árboles de tamaño grande, salvo en las orillas de los ríos grandes y en los manchones del BTC. Esta situación ha sido registrada en otros trabajos con condiciones similares (García-Vázquez et al., 2006; Ramírez-Bautista, 1994). Asimismo, la escasez de cuerpos de agua en gran parte de la zona árida limita la presencia de un mayor número de especies con hábitos acuáticos, las cuales se encuentran únicamente en la vega de los ríos, arroyos y canales de riego, o en algunos cuerpos de agua permanentes. Algunos ejemplos de estas especies son: Ambystoma velasci y Nerodia rhombifer.

En lo referente a los atributos de historia natural de los organismos, se encontró que la mayoría de las especies de anfibios son nocturnas, que de acuerdo con algunos autores puede ser una adaptación para combatir las temperaturas altas y la humedad baja (García-Vázquez et al., 2006). Estas condiciones climáticas, sobre todo la humedad baja, provoca que la riqueza de anfibios de la ZAHGO (20 sp.) sea mucho menor que en zonas con un mayor grado de humedad, como son los bosques mesófilos de montaña (33 sp.), ya que la humedad es un requerimiento indispensable para los anfibios (Ramírez-Bautista et al., 2014). Para el caso de los saurópsidos no aves, la falta de humedad no es una limitante, por el contrario, tienen mayor resistencia a cambios ambientales (Suazo-Ortuño, Alvarado-Díaz y Martínez-Ramos, 2008), y los escamados son un grupo bien representado en las zonas desérticas (Fernández-Badillo, 2013; Flores-Villela y Goyenechea, 2003). Respecto a los patrones de actividad, la mayoría de las lagartijas presentan actividad diurna, lo cual ha sido observado en otras regiones cálidas (García-Vázquez et al., 2006), en tanto que la mayoría de las serpientes presentan actividad nocturna, lo cual se debe a diversas razones, por ejemplo, para sincronizar su actividad con la de sus presas, para disminuir el riesgo de ser depredadas al estar activas en las horas de menor actividad de sus depredadores, para aprovechar temperaturas óptimas durante la noche o evitar las horas más calientes del día (Sperry, Ward y Weatherhead, 2013).

En cuanto al tipo de alimentación, la mayor parte de los anfibios como de los saurópsidos no aves de las zonas áridas de Hidalgo presentaron una preferencia por los insectos y artrópodos en general. Dado que la mayor parte de las especies presentes en estas zonas son de tamaño pequeño, también lo son sus presas, pues en varios trabajos se ha encontrado una relación entre el tamaño de las presas y el tamaño del cuerpo de anfibios y saurópsidos no aves (Fatroandrianjafinonjasolomiovazo, Rasoamampionona, Vieites y Vences, 2011; Kaliontzopoulou, Adams, van der Meijden, Perera y Carretero, 2012; Ngo, Ngo, Truong y Duong, 2014). Características como el tamaño de la cabeza y ancho del hocico provocan diferencias en el consumo de presas, incluso entre individuos de clases de edad y sexos distintos de la misma especie (Kaliontzopoulou et al., 2012; Ngo et al., 2014). En contraste con esto, la mayor parte de las serpientes consume presas como vertebrados pequeños, roedores e incluso mamíferos de tamaño mayor.
Con respecto a los tipos de paridad, se encontró que todas las especies de anfibios que habitan en la ZAHGO son ovíparas, ya que la viviparidad es una característica extremadamente rara en este grupo de vertebrados. Aunque la viviparidad se ha originado en los 3 órdenes de anfibios actuales, la proporción de especies de anuros y urodelos con este tipo de paridad, es extremadamente baja (Vitt y Caldwell, 2014; Wake, 1993). En el caso de los saurópsidos no aves, la mayor parte de las especies (37) son ovíparas. Dentro de los saurópsidos, el tipo de paridad ancestral es la ovoparidad, y la viviparidad es exclusiva de algunos integrantes del orden Squamata (Shine, 1995; Stewart y Blackburn, 2014). En estos, la viviparidad es predominante en especies de zonas templadas de altitudes y latitudes altas (Shine, 1995), pero las ventajas que ofrece la retención de los embriones en oviducto ha permitido que invadan zonas tropicales y ocupen hábitats tan diversos como selvas, desiertos y ambientes xéricos (Shine, 1995; Stewart y Blackburn, 2014).

La presencia de un gran número de especies endémicas para México de anfibios y saurópsidos no aves en la zona árida de Hidalgo, aunada a su riqueza específica, mucho mayor que la registrada en trabajos previos (Camarillo-Rangel, 1993; Hernández-Salinas y Ramírez-Bautista, 2012, 2013; RamírezBautista et al., 2010, 2014), la cual únicamente se ve superada por el bosque mesófilo de montaña (93 sp.), que es el tipo de vegetación con los valores de riqueza de herpetozoos más alta del estado (Ramírez-Bautista et al., 2014), le confiere a esta región una gran importancia en términos de conservación, ya que, tanto por su ubicación geográfica, sus condiciones ambientales y su historia biogeográfica, le permiten albergar una herpetofauna integrada por una mezcla de especies muy particular. Sin embargo, al igual que la mayoría de las zonas áridas del país, la ZAHGO se ve altamente afectada por la acelerada perturbación a causa de las actividades humanas, como la sobreexplotación de la cobertura vegetal, la erosión del suelo, el sobrepastoreo (Rzedowski, 2006) y el establecimiento de zonas de cultivo. Además, las poblaciones de serpientes en general, así como las poblaciones de las lagartijas del género Gerrhonotus, se ven afectadas por la intensa persecución humana (Fernández-Badillo y Goyenechea, 2010), debido a que los pobladores las aniquilan en cuanto son observadas. Actualmente, se cuenta con una ANP (Reserva de la Biosfera Barranca de Metztitlán), localizada en la parte norte de la ZAHGO, en la ecorregión del MMC, la cual constituye el ANP más grande del estado (Conanp, 2003). La ecorregión del MCM por su parte no cuenta con áreas para la protección de la biodiversidad y dadas las diferencias encontradas en la composición de especies antes mencionadas, sería importante proteger alguna zona de esta ecorregión. Un sitio probable para el establecimiento de un área protegida podría ubicarse en la porción oriental del Valle del Mezquital, la cual presenta una gran riqueza herpetofaunística (Fernández-Badillo y Goyenechea, 2010) y que ha sido sugerida anteriormente como una zona prioritaria de conservación, debido tanto a su diversidad herpetológica como a su geodiversidad (Lara-Parra, 2014). Aunque debido a las actividades productivas que se realizan en la región, como la explotación del maguey, el sobrepastoreo, la tala inmoderada, el grado de perturbación antrópica derivada de la fragmentación del hábitat y el crecimiento de los 
asentamientos humanos (Moreno, Garret y Fierro, 2006) que complican las posibles propuestas de conservación, el Valle del Mezquital constituye un área de gran importancia ecológica, económica, social, histórica y cultural (López-Galindo, 2001), por lo que el establecimiento de zonas de conservación, así como sos, permitiría tanto la preservación de las especies, como una alternativa económica a los pobladores de la región. de proyectos para el aprovechamiento sustentable de los recur-

\section{Agradecimientos}

Los autores agradecen al proyecto «Diversidad Biológica del Estado de Hidalgo (tercera etapa) Fomix-Conacyt-HGO-2012191908» por el apoyo para realizar el estudio, así como a los 2 revisores anónimos, que con sus comentarios enriquecieron el manuscrito.

\section{Anexo.}

Relación de especies y referencias bibliográficas para la herpetofauna de la zona árida de Hidalgo: 1) Altamirano-Álvarez, Mendoza-Quijano, Marmolejo-Santillán y García-Collazo, 1993; 2) Camarillo-Rangel, 1993; 3) Campos-Rodríguez et al., 2010; 4) Fernández-Badillo, 2008; 5) Fernández-Badillo y Goyenechea, 2010; 6) Gelover-Alfaro et al., 2000; 7) Gómez-Mendoza, 2007; 8) Goyenechea et al., 2010; 9) Hernández-Pérez, 1997; 10) Hernández-Salinas, 2009; 11) Huitzil-Mendoza, 2010; 12) Lara-Parra, 2014; 13) López-Mejía, 2011; 14) Martín del Campo, 1936; 15) Martín del Campo, 1937; 16) Mendoza-Quijano, 1990;

17) Mendoza-Quijano y Smith, 1993; 18) Morales-Capellán, 2010; 19) Ramírez-Bautista et al., 2010; 20) Ramírez-Bautista et al., 2014; 21) Roth-Monzón, 2010; 22) Vite-Silva, 2008, y 23) Vite-Silva et al., 2010.

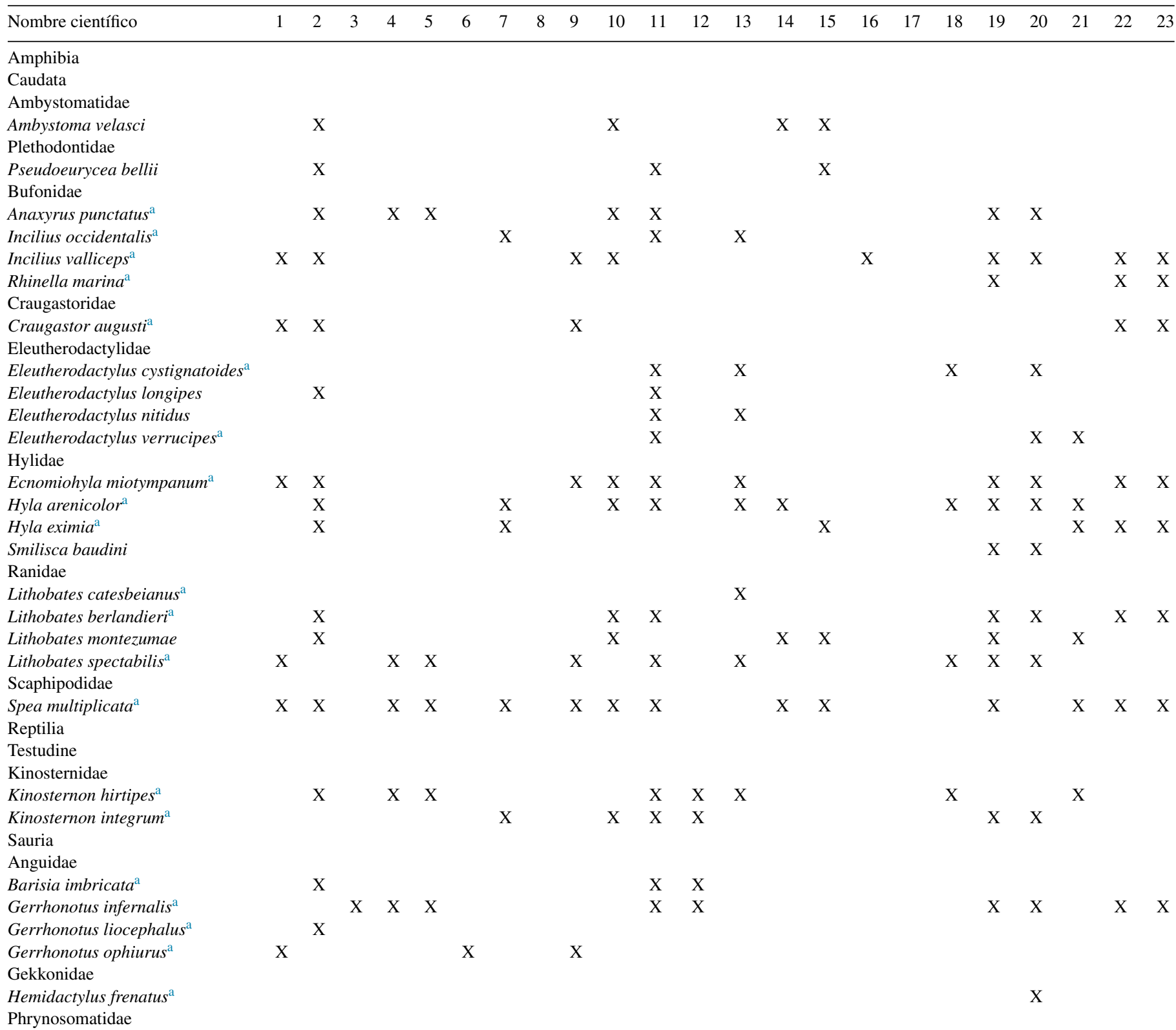




\begin{tabular}{|c|c|c|c|c|c|c|c|c|c|c|c|c|c|c|c|c|c|c|c|c|c|c|c|}
\hline Nombre científico & 1 & 2 & 3 & 4 & 5 & 6 & 7 & 8 & 9 & 10 & 11 & 12 & 13 & 14 & 15 & 16 & 17 & 18 & 19 & 20 & 21 & 22 & 23 \\
\hline $\begin{array}{l}\text { Phrynosoma } \\
\text { orbiculare }^{\mathrm{a}}\end{array}$ & & $X$ & & & & & & & & $X$ & $\mathrm{X}$ & $X$ & & $X$ & $X$ & & & & $X$ & $X$ & & $X$ & $X$ \\
\hline $\begin{array}{l}\text { Sceloporus } \\
\quad \text { grammicus }^{\mathrm{a}}\end{array}$ & & & & & & & $X$ & & & & $X$ & $\mathrm{X}$ & & $X$ & & & & & $X$ & $\mathrm{X}$ & & $X$ & $\mathrm{X}$ \\
\hline $\begin{array}{l}\text { Sceloporus } \\
\quad \text { megalepidurus }^{\mathrm{a}}\end{array}$ & & & & & & & & & & & & & & & & & & & & $X$ & & & \\
\hline Sceloporus minor ${ }^{\mathrm{a}}$ & $X$ & $\mathrm{X}$ & & $X$ & $\mathrm{X}$ & $X$ & & & $X$ & $\mathrm{X}$ & $\mathrm{X}$ & $\mathrm{X}$ & $X$ & & & $X$ & & & $\mathrm{X}$ & $X$ & & $\mathrm{X}$ & $\mathrm{X}$ \\
\hline $\begin{array}{l}\text { Sceloporus } \\
\quad \text { mucronatus }^{\mathrm{a}}\end{array}$ & & & & & & & & & & $\mathrm{X}$ & & $\mathrm{X}$ & & & & & & & $\mathrm{X}$ & & $X$ & $\mathrm{X}$ & $X$ \\
\hline Sceloporus parvus ${ }^{\mathrm{a}}$ & & $X$ & & $X$ & $X$ & & & & & $X$ & $X$ & $X$ & & & $X$ & $X$ & & & $X$ & $X$ & & $X$ & $X$ \\
\hline Sceloporus spinosus ${ }^{\mathrm{a}}$ & $X$ & $\mathrm{X}$ & & $X$ & $\mathrm{X}$ & $X$ & $X$ & & $X$ & $\mathrm{X}$ & $\mathrm{X}$ & $\mathrm{X}$ & $X$ & $X$ & $\mathrm{X}$ & $X$ & & $X$ & $\mathrm{X}$ & $\mathrm{X}$ & $X$ & $\mathrm{X}$ & $\mathrm{X}$ \\
\hline Sceloporus torquatus ${ }^{\mathrm{a}}$ & & $\mathrm{X}$ & & & & & $X$ & & & $\mathrm{X}$ & $X$ & $\mathrm{X}$ & & $X$ & $X$ & & & & $\mathrm{X}$ & & $X$ & $\mathrm{X}$ & $\mathrm{X}$ \\
\hline $\begin{array}{l}\text { Sceloporus variabilis }{ }^{\mathrm{a}} \\
\text { Scincidae }\end{array}$ & $X$ & $\mathrm{X}$ & & & & $\mathrm{X}$ & & & $X$ & $\mathrm{X}$ & $\mathrm{X}$ & $\mathrm{X}$ & $\mathrm{X}$ & & & $X$ & & $X$ & $\mathrm{X}$ & $X$ & & $\mathrm{X}$ & $\mathrm{X}$ \\
\hline Plestiodon lynxe ${ }^{\mathrm{a}}$ & & $X$ & & & & & & & & & & & & & & & & & $\mathrm{X}$ & & & $X$ & $X$ \\
\hline $\begin{array}{l}\text { Scincella } \\
\quad \text { gemmingeri }^{\mathrm{a}}\end{array}$ & $X$ & & $X$ & & & $X$ & & & $X$ & & & $\mathrm{X}$ & & & & & & & $X$ & & & $X$ & $X$ \\
\hline Teiidae & & & & & & & & & & & & & & & & & & & & & & & \\
\hline $\begin{array}{l}\text { Aspidoscelis gularis }^{\mathrm{a}} \\
\text { Xantusiidae }\end{array}$ & $\mathrm{X}$ & $\mathrm{X}$ & & $X$ & $\mathrm{X}$ & $\mathrm{X}$ & $X$ & & $X$ & $\mathrm{X}$ & $\mathrm{X}$ & $\mathrm{X}$ & $X$ & $\mathrm{X}$ & $\mathrm{X}$ & $X$ & & $\mathrm{X}$ & $X$ & $\mathrm{X}$ & $X$ & $\mathrm{X}$ & $\mathrm{X}$ \\
\hline Lepidophyma gaigeae & & & & & & & & & & & $X$ & $X$ & $X$ & & & & & & & & & & \\
\hline $\begin{array}{l}\text { Lepidophyma occulor } \\
\text { Serpentes }\end{array}$ & $X$ & & & & & $\mathrm{X}$ & & & $\mathrm{X}$ & & & & & & & & & & & & & $X$ & $X$ \\
\hline Boidae & & & & & & & & & & & & & & & & & & & & & & & \\
\hline $\begin{array}{l}\text { Boa imperator }{ }^{\mathrm{a}} \\
\text { Colubridae }\end{array}$ & & & & & & & & $X$ & & & & & & & & & & & & & & & \\
\hline Conopsis lineata $^{\mathrm{a}}$ & & $\mathrm{X}$ & & & & & & & & $\mathrm{X}$ & $X$ & $\mathrm{X}$ & & & & & & & $X$ & & $X$ & $X$ & $\mathrm{X}$ \\
\hline Conopsis nasus & & & & & & & & & & & & $\mathrm{X}$ & & & & & & & & & $\mathrm{X}$ & & \\
\hline $\begin{array}{l}\text { Drymarchon } \\
\text { melanurus }^{\mathrm{a}}\end{array}$ & $X$ & $X$ & $\mathrm{X}$ & $X$ & $X$ & $X$ & & & $X$ & & $X$ & $\mathrm{X}$ & $X$ & & $X$ & $X$ & & $X$ & $X$ & & & $X$ & $X$ \\
\hline Ficimia hardyi ${ }^{\mathrm{a}}$ & & & & $\mathrm{X}$ & $\mathrm{X}$ & & & & & & & & & & & & $X$ & & & & & $\mathrm{X}$ & $\mathrm{X}$ \\
\hline $\begin{array}{l}\text { Lampropeltis } \\
\text { mexicana }^{\text {a }}\end{array}$ & & & & & & & $X$ & & & & & & & & & & & & & & & & \\
\hline $\begin{array}{l}\text { Lampropeltis } \\
\text { polyzona }^{\text {a }}\end{array}$ & & & & & & & & & & & & & & & & & & & & $X$ & & & \\
\hline Lampropeltis ruthveni & & & & & & & & & & & & & & & & & & & & & $X$ & & \\
\hline Masticophis schotti ${ }^{\mathrm{a}}$ & & $X$ & & $X$ & $X$ & & $X$ & & & $X$ & $X$ & $X$ & $X$ & $X$ & & $X$ & & $\mathrm{X}$ & & & $\mathrm{X}$ & $\mathrm{X}$ & $\mathrm{X}$ \\
\hline Oxybelis aeneus & $X$ & & & & & $X$ & & & $X$ & & & & & & & & & & & $X$ & & & \\
\hline Pantherophis emoryi ${ }^{\mathrm{a}}$ & & & & $X$ & $\mathrm{X}$ & & & & & & $\mathrm{X}$ & $X$ & & & & & & & $X$ & & & & \\
\hline Pituophis deppe $\mathrm{i}^{\mathrm{a}}$ & & & & $\mathrm{X}$ & $\mathrm{X}$ & & $X$ & & & $X$ & $\mathrm{X}$ & $\mathrm{X}$ & $X$ & $X$ & & & & $X$ & $\mathrm{X}$ & $X$ & X & $X$ & $X$ \\
\hline Rhadinaea gaigea $^{\mathrm{a}}$ & & & & & & & & & & & & & & & & & & & $\mathrm{X}$ & & & & \\
\hline Salvadora bairdi $i^{\mathrm{a}}$ & & & & & & & $X$ & & & & $\mathrm{X}$ & $\mathrm{X}$ & & & & & & $X$ & & $\mathrm{X}$ & $X$ & & \\
\hline $\begin{array}{l}\text { Salvadora } \\
\quad \text { grahamiae }^{\mathrm{a}}\end{array}$ & & & & $\mathrm{X}$ & $\mathrm{X}$ & & & & & $\mathrm{X}$ & $\mathrm{X}$ & & & & & & & & & $\mathrm{X}$ & & & \\
\hline Senticolis triaspis ${ }^{\mathrm{a}}$ & $X$ & $X$ & & & & $X$ & & & $X$ & & $X$ & & $X$ & & & & & & & & & $X$ & $\mathrm{X}$ \\
\hline Tantilla bocourti $i^{\mathrm{a}}$ & & $\mathrm{X}$ & & $X$ & $X$ & & & & & & $\mathrm{X}$ & $X$ & & & & & & $X$ & & & & & \\
\hline Trimorphodon tau ${ }^{\mathrm{a}}$ & & $\mathrm{X}$ & $\mathrm{X}$ & $\mathrm{X}$ & $\mathrm{X}$ & & & & & & $\mathrm{X}$ & $\mathrm{X}$ & $\mathrm{X}$ & & & & & & $X$ & & & $\mathrm{X}$ & $\mathrm{X}$ \\
\hline $\begin{array}{l}\text { Tropidodipsas } \\
\text { sartorii }^{\mathrm{a}}\end{array}$ & & $\mathrm{X}$ & $\mathrm{X}$ & $\mathrm{X}$ & $\mathrm{X}$ & & & & & $\mathrm{X}$ & $\mathrm{X}$ & $\mathrm{X}$ & & & & & & $\mathrm{X}$ & & & & & \\
\hline Dipsadidae & & & & & & & & & & & & & & & & & & & & & & & \\
\hline Diadophis punctatus & & & & & & & & & & $\mathrm{X}$ & & & & & & & & & & & & & \\
\hline Geophis latifrontalis $^{\mathrm{a}}$ & & & & & & & & & & & $X$ & & & & & & & & & & & $X$ & $\mathrm{X}$ \\
\hline Hypsiglena jani $^{\mathrm{a}}$ & & $\mathrm{X}$ & & $X$ & $\mathrm{X}$ & & & & & $X$ & $\mathrm{X}$ & $\mathrm{X}$ & $\mathrm{X}$ & & & & & $X$ & & & & & \\
\hline Leptodeira maculata & $\mathrm{X}$ & & $X$ & & & $X$ & & & $X$ & & & & & & & & & & & & & & \\
\hline $\begin{array}{l}\text { Leptodeira } \\
\quad \text { septentrionalis }^{\mathrm{a}}\end{array}$ & $\mathrm{X}$ & & & & & $\mathrm{X}$ & & & $\mathrm{X}$ & & & $\mathrm{X}$ & & & & $\mathrm{X}$ & & & & & & $X$ & $\mathrm{X}$ \\
\hline Leptophis mexicanus & & & & & & & & & & & $\mathrm{X}$ & & & & & & & & $\mathrm{X}$ & $X$ & & & \\
\hline Storeria dekayi $i^{\mathrm{a}}$ & & & & & & & & & & & & & & & & & & & & & & $\mathrm{X}$ & $\mathrm{X}$ \\
\hline $\begin{array}{l}\text { Storeria hidalgoensis } \\
\text { Elapidae }\end{array}$ & & & & & & & & & & & & $\mathrm{X}$ & & & & & & & & & & & \\
\hline $\begin{array}{l}\text { Micrurus tener } \\
\text { Leptotyphlopidae }\end{array}$ & & $\mathrm{X}$ & & $X$ & $X$ & & & & & & $X$ & $\mathrm{X}$ & & & & $X$ & & & $X$ & $\mathrm{X}$ & & $\mathrm{X}$ & $X$ \\
\hline $\begin{array}{l}\text { Rena dulcis }^{\mathrm{a}} \\
\text { Natricidae }\end{array}$ & $X$ & $\mathrm{X}$ & $\mathrm{X}$ & $X$ & $X$ & $X$ & & & $X$ & $X$ & $X$ & $\mathrm{X}$ & & & & & & $X$ & $X$ & & & $X$ & $X$ \\
\hline
\end{tabular}




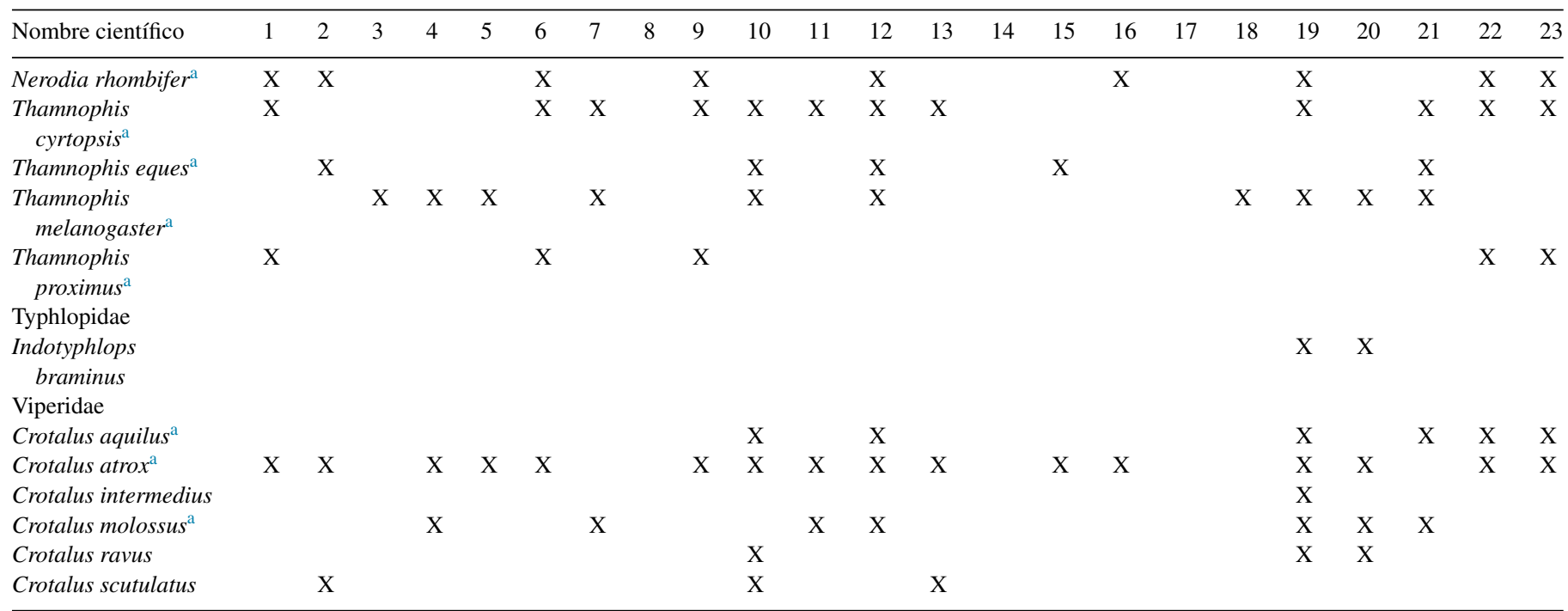

a Especies observadas en el presente estudio.

\section{Referencias}

Altamirano-Álvarez, T., Mendoza-Quijano, F., Marmolejo-Santillán, Y. y García-Collazo, R. (1993). Senticolis triaspis intermedius (Boetger), Colubridae, un nuevo registro para el estado de Hidalgo, México; comentarios en sus distribución. Boletín de la Sociedad Herpetológica Mexicana, 5, 6-8.

Bryson, R. W., Jr., Jaeger, J. R., Lemos-Espinal, J. A. y Lazcano, D. (2012). A multilocus perspective on the speciation history of a North American aridland toad (Anaxyrus punctatus). Molecular Phylogenetics and Evolution, 64, 393-400.

Calzada-Arciniega, R. A. y Hernández-Ríos, A. (2014). Anaxyrus punctatus. Geographic distribution. Herpertological Review, 45, 276.

Camarillo-Rangel, J. L. (1993). Algunos aspectos biogeográficos de los anfibios y reptiles de la zona xerófila de Hidalgo. En M. A. Villavicencio, Y. Marmolejo-Santillán y B. E. Pérez (Eds.), Investigaciones recientes sobre flora y fauna de Hidalgo, México (pp. 415-432). Pachuca, Hidalgo: Centro de Investigaciones Biológicas, División de Investigación, Universidad Nacional Autónoma de Hidalgo.

Camarillo-Rangel, J. L. (2002). Observaciones en la distribución de Oxybelis aeneus (Squamata: Colubridae). Boletín de la Sociedad Herpetológica Mexicana, 10, 13-14.

Campbell, J. A. y Lamar, W. W. (2004). The venomous reptiles of the Western hemisphere. Ithaca, New York: Comstock.

Campos-Rodríguez, J. I. y Mendoza-Quijano, F. (2004). Crotalus ravus. Geographic distribution. Herpetological Review, 35, 291.

Campos-Rodríguez, J. I., Pérez-Valera, B. y Flores-Leyva, X. (2010). Reptiles notables en la región semiárida del estado de Hidalgo. Boletín de la Sociedad Herpetológica Mexicana, 18, 1-10.

Canseco-Márquez, L., Mendoza-Quijano, F. y Gutiérrez-Mayén, M. G. (2004). Análisis de la distribución de la herpetofauna. En I. Luna-Vega, J. J. Morrone, y D. Esparza (Eds.), Biodiversidad de la Sierra Madre Oriental (p. 417338). México, D.F.: Comisión Nacional para el Conocimiento y Uso de la Biodiversidad. Universidad Nacional Autónoma de México.

Canseco-Márquez, L. y Gutiérrez-Mayén, M. G. (2010). Anfibios y reptiles del Valle de Tehuacán-Cuicatlán. Comisión Nacional para el Conocimiento y Uso de la Biodiversidad. Puebla: Fundación para la Reserva de la Biosfera Cuicatlán, A.C., Benemérita Universidad Autónoma de Puebla.

Colwell, R. K. y Coddington, J. A. (1994). Estimating terrestrial biodiversity trough exploration. Philosophical Transactions of the Royal Society London $B, 345,101-118$.

Conanp (Comisión Nacional de Áreas Naturales Protegidas). (2003). Programa de manejo de la Reserva de la Biosfera Barranca de Metztitlán, México. México, D.F.: Comisión Nacional de Áreas Naturales Protegidas.
Dixon, J. R. y Lemos-Espinal, J. A. (2010). Anfibios y reptiles del estado de Querétaro, México. México, D.F.: Texas A y M University, Universidad Nacional Autónoma de México y Comisión Nacional para el Conocimiento y Uso de la Biodiversidad.

Espinosa-Organista, D. y Ocegueda, S. (2008). El conocimiento biogeográfico de las especies y su regionalización natural. En Capital natural de México. Vol. I. Conocimiento actual de la biodiversidad. pp. 33-65. México, D.F.: Comisión Nacional para el Conocimiento y Uso de la Biodiversidad.

ESRI (Environmental Systems Research Institute). (1998). ArcViewGIS 3.2. Redlands, California: Environmental Systems Research Institute.

Fatroandrianjafinonjasolomiovazo, T. N. L., Rasoamampionona, N. R., Vieites, D. R. y Vences, V. (2011). Diet of the Mascarene grass frog, Ptychadena mascareniensis, in Madagascar. Malagasy Nature, 5, 68-74.

Fernández, J., Sánchez, O. y Flores-Villela, O. (2006). Anfibios y reptiles del estado de Tlaxcala. En A. Ramírez-Bautista, L. Canseco-Márquez y F. Mendoza-Quijano (Eds.), Inventarios herpetofaunísticos de México. Avances en el conocimiento de su biodiversidad (pp. 224-240). México, D.F.: Publicaciones de la Sociedad Herpetológica Mexicana.

Fernández-Badillo, L. (2008). Anfibios y reptiles del Alto Mezquital, Hidalgo. Tesis. Facultad de Estudios Superiores Iztacala, Universidad Nacional Autónoma de México.

Fernández-Badillo, L. (2013). Patrones de distribución de las lagartijas y las serpientes en los desiertos mexicanos. Tesis de maestría. Ciencias en Biodiversidad y Conservación. Universidad Autónoma del Estado de Hidalgo.

Fernández-Badillo, L. y Goyenechea, I. (2010). Anfibios y reptiles del valle del Mezquital, Hidalgo, México. Revista Mexicana de Biodiversidad, 81, 705-712.

Fernández-Badillo, L., Morales-Capellán, N. y Goyenechea, I. (2011). Serpientes venenosas del estado de Hidalgo. Pachuca: Universidad Autónoma del Estado de Hidalgo.

Flores-Villela, O. y Canseco-Márquez, L. (2004). Nuevas especies y cambios taxonómicos para la herpetofauna de México. Acta Zoológica Mexicana (nueva serie), 20, 115-144.

Flores-Villela, O. y Goyenechea, I. (2003). Patrones de distribución de anfibios y reptiles en México. En J. J. Morrone y J. Llorente-Bousquets (Eds.), Una perspectiva latinoamericana de la biogeografía (pp. 289-296). México, D.F.: Las Prensas de Ciencias, Facultad de Ciencias, Universidad Nacional Autónoma de México.

García-Vázquez, U. O., Canseco-Márquez, L., Aguilar-López, J. L., HernándezJiménez, C. A., Maceda-Cruz, J., Gutiérrez-Mayén, M. G., et al. (2006). Análisis de la distribución de la herpetofauna en la región Mixteca de Puebla, México. En A. Ramírez-Bautista, L. Canseco-Márquez y F. MendozaQuijano (Eds.), Inventarios herpetofaunísticos de México. Avances en el 
conocimiento de su biodiversidad (pp. 152-169). México, D.F.: Publicaciones de la Sociedad Herpetológica Mexicana.

Gelover-Alfaro, A., Altamirano-Álvarez, T. y Soriano, M. (2000). Uso de los recursos espacio-temporales de la herpetofauna de Metztitlán, Hidalgo. Revista Zoológica, 11, 4-11.

Gómez-Mendoza, J. (2007). Contribución al conocimiento de la herpetofauna del municipio de Tepeji del Río de Ocampo, Hidalgo. Tesis. Facultad de Estudios Superiores Iztacala, Universidad Nacional Autónoma de México.

Goyenechea, I., Morales-Capellán, N. y Fernández-Badillo, L. (2011a). Crotalus molossus (Black-tailed Rattlesnake). Geographic distribution. Herpetological Review, 42, 571-572.

Goyenechea, I., Morales-Capellán, N. y Fernández-Badillo, L. (2011b). Crotalus atrox (Western Diamond-backed Rattlesnake). Geographic distribution. Herpetological Review, 42, 571

Goyenechea, I., Morales-Capellán, N. y Fernández-Badillo, L. (2011c). Crotalus aquilus (Qureteran Dusky Rattlesnake). Geographic distribution. Herpetological Review, 42, 571.

Goyenechea, I., Vite, V. D. y Mendiola-González, M. E. (2010). Boa constrictor. Geographic distribution. Herpetological Review, 41, 515.

Granados-Sánchez, D., Sánchez-Gonzáles, A., Granados-Victorino, R. L. y Borja-de la Rosa, A. (2011). Ecología de la vegetación del desierto chihuahuense. Revista Chapingo Serie Ciencias Forestales y del Ambiente, XVII, $111-130$.

Hernández, H. M. (2006). La vida en los desiertos mexicanos. México, D.F.: Fondo de Cultura Económica.

Hernández-Ibarra, X. y Ramírez-Bautista, A. (2006). Herpetofauna del municipio de Guadalcázar, San Luis Potosí, México. En A. Ramírez-Bautista, L. Canseco-Márquez y F. Mendoza-Quijano (Eds.), Inventarios herpetofaunísticos de México. Avances en el conocimiento de su biodiversidad (pp. 58-73). México, D.F.: Publicaciones de la Sociedad Herpetológica Mexicana.

Hernández-Pérez, E. (1997). La herpetofauna de Metztitlán, Hidalgo, México: problemática e importancia. Tesis. Escuela Nacional de Estudios Profesionales Iztacala, Universidad Nacional Autónoma de México.

Hernández-Salinas, U. (2009). Estudio herpetofaunístico del estado de Hidalgo, México. Tesis de maestría. Universidad Autónoma del Estado de Hidalgo.

Hernández-Salinas, U., Cruz-Elizalde, R., Berriozábal-Islas, C. y Goyenechea, I. (2011). Rena (Leptoptyphlops) dulcis. Geographic distribution. Herpetological Review, 42, 244.

Hernández-Salinas, U. y Ramírez-Bautista, A. (2010). Ramphotyphlops braminus (Brahminy Blind Snake). Geographic distribution. Herpetological Review, 41, 519 .

Hernández-Salinas, U. y Ramírez-Bautista, A. (2012). Diversity of amphibian communities in four vegetation types of Hidalgo State, Mexico. The Open Conservation Biology Journal, 6, 1-11.

Hernández-Salinas, U. y Ramírez-Bautista, A. (2013). Distribución de la herpetofauna en cuatro tipos de vegetación del estado de Hidalgo, México. En G. Pulido-Flores y S. Monks (Eds.), Estudios científicos en el estado de Hidalgo y zonas aledañas, Vol. II (pp. 5-12). Lincoln, Nebraska: Zea-E-Books.

Huitzil-Mendoza, J. C. (2007). Herpetofauna de dos localidades en la región norte de Zimapán, Hidalgo. Tesis. Universidad Autónoma del Estado de Hidalgo.

Huitzil-Mendoza, J. C. (2010). Anfibios y reptiles de la zona árida al noreste del estado del Hidalgo. Tesis de maestría. Universidad Autónoma del Estado de Hidalgo.

Huitzil-Mendoza, J. C. y Goyenechea, I. (2011). Primeros registros de herpetozoos en Zimapán, Hidalgo, México. Revista Mexicana de Biodiversidad, $82,717-720$.

Kaliontzopoulou, A., Adams, D. C., van der Meijden, A., Perera, A. y Carretero, M. A. (2012). Relationships between head morphology, bite performance and ecology in two species of Podarcis wall lizards. Evolutionary Ecology, 26, 825-845.

Lara-Parra, A.I. (2014). Diversidad de saurópsidos en el Valle del Mezquital, México, un enfoque de conservación de áreas naturales con base en la geodiversidad. Tesis de maestría. Universidad Autónoma del Estado de Hidalgo.

Lemos-Espinal, J. A. y Dixon, J. R. (2013). Amphibians and reptiles of San Luis Potosí. Utah: Eagle Mountain Publishing, L.C.
Lemos-Espinal, J.A. y Smith, H.M. (2008). Anfibios y reptiles del estado de Chihuahua, México. Universidad Nacional Autónoma de México. México, D.F.: University of Colorado at Boulder y Comisión Nacional para el Conocimiento y Uso de la Biodiversidad.

López-Galindo, F. (2001). Evaluación y planeación ecológica del uso de suelo, en los municipios de Cardonal, Tasquillo y norte de Ixmiquilpan, Hidalgo. Tesis de maestría. Facultad de Ciencias. Universidad Nacional Autónoma de México.

López-Mejía, A. (2011). Herpetofauna de la localidad de la Paila, municipio de Tecozautla, Hidalgo. Tesis. Universidad Autónoma del Estado de Hidalgo.

López-Mejía, A. y Goyenechea, I. (2012a). Lepidophyma gaigeae (Gaige's Tropical Night Lizard). Geographic distribution. Herpetological Review, 43, 306.

López-Mejía, A. y Goyenechea, I. (2012b). Lithobates spectabilis (Showy Leopard Frog). Geographic distribution. Herpetological Review, 43, 300.

López-Mejía, A. y Goyenechea, I. (2012c). Incilius occidentalis (Pine Toad). Geographic distribution. Herpetological Review, 43, 299.

López-Mejía, A. y Goyenechea, I. (2012d). Eleutherodactylus nitidus (Shiny Peeping Frog). Herpetological Review, 43, 298.

Marmolejo-Santillán, Y. (1991). Estado del conocimiento de la fauna silvestre del Valle del Mezquital, Hidalgo, México. Pancromo, 23, 12-18

Martín del Campo, R. (1936). Contribución al conocimiento de la fauna de Actopan, Hidalgo. IV. Vertebrados observados en la época de seca. Anales del Instituto de Biología, Univerisdad Nacional Autónoma de México, 7 , 271-278.

Martín del Campo, R. (1937). Contribución al conocimiento de los batracios y reptiles del Valle del Mezquital, Hidalgo. Anales del Instituto de Biología, Univerisdad Nacional Autónoma de México, 8, 260-266.

Martínez-Morales, M. A., Ortiz-Pulido, R., de la Barreda, B., Zuria, I. L., BravoCadena, J. y Valencia-Herverth, J. (2007). Hidalgo. En R. Ortiz-Pulido, A. Navarro-Sigüenza, H. Gómez de Silva, O. Rojas-Soto y T. A. Peterson (Eds.), Avifaunas estatales de México (pp. 49-95). Pachuca: CIPAMEX.

Mendoza-Quijano, F. (1990). Estudio herpetofaunístico en el transecto Zacualtipán- Zoquizoquipan-San Juan Metztitlán, Hidalgo. Tesis. Escuela Nacional de Estudios Profesionales Iztacala, Universidad Nacional Autónoma de México.

Mendoza-Quijano, F. y Smith, H. M. (1993). A new species of Hooknose Snake, Ficimia (Reptilia, Serpentes). Journal of Herpetology, 27, 406-410.

Miranda, F. y Hernández, X. E. (1963). Los tipos de vegetación en México y su clasificación. Boletín de la Sociedad Botánica Mexicana, 28, 27-72.

Morales-Capellán, N. (2010). Herpetofauna de dos ambientes contrastantes del municipio de Tasquillo, Hidalgo. Tesis. Universidad Autónoma del Estado de Hidalgo.

Morales-Capellán, N., Fernández-Badillo, L. y Goyenechea, I. (2011). Senticolis triaspis (Green Rat Snake). Geographic distribution. Herpetological Review, 42,574 .

Moreno, C. (2001). Métodos para medir la biodiversidad. Zaragoza: MyTManuales y Tesis SEA.

Moreno, B., Garret, G. y Fierro, U. (2006). Otomíes del Valle del Mezquital. México, D.F.: Comisión para el Desarrollo de los Pueblos Indígenas.

Morrone, J. J. (2005). Hacia una síntesis biogeográfica de México. Revista Mexicana de Biodiversidad, 76, 207-252.

Ngo, C. D., Ngo, B. V., Truong, P. B. y Duong, L. D. (2014). Sexual size dimorphism and feeding ecology of Eutropis multifasciata (Reptilia: Squamata: Scincidae) in the central highlands of Vietnam. Herpetological Conservation and Biology, 9, 322-333.

Nieto-Montes de Oca, A. (1999). Anfibios y reptiles del estado de Querétaro. Informe final del proyecto H250. México, D.F.: Comisión Nacional para el Conocimiento y Uso de la Biodiversidad.

Olson, D. M., Dinerstein, E., Wikramanayake, E. D., Burges, N. D., Powell, G. V. N., Underwood, E. C., et al. (2001). Terrestrial ecoregions of the world: a new map of life on earth (PDF, 1. $1 \mathrm{M}$ ). BioScience, 51, 933-938.

Pampa-Ramírez, J. T. (2010). Revisión taxonómica de las especies de la familia Leptotyphlopidae para el estado de Hidalgo. Tesis. Universidad de Guadalajara.

Ramírez-Bautista, A. (1994). Manual y claves ilustradas de los anfibios y reptiles de la región de Chamela, Jalisco. México. Cuadernos del Instituto de Biología No. 28. México, D.F.: Universidad Nacional Autónoma de México. 
Ramírez-Bautista, A., Hernández-Salinas, U., Cruz-Elizalde, R., BerriozabalIslas, C., Lara-Tufiño, D., Goyenechea, I., et al. (2014). Los anfibios y reptiles del estado de Hidalgo, México: diversidad, biogeografía y conservación. Pachuca, Hidalgo: Sociedad Herpetológica Mexicana.

Ramírez-Bautista, A., Hernández-Salinas, U., García-Vázquez, U. O., LeyteManrique, A. y Canseco-Márquez, L. (2009). Herpetofauna del Valle de México: diversidad y conservación. México, D.F.: Universidad Autónoma del Estado de Hidalgo y Comisión Nacional para el Conocimiento y Uso de la Biodiversidad.

Ramírez-Bautista, A., Hernández-Salinas, U., Mendoza-Quijano, F., CruzElizalde, R., Stephenson, B. P., Vite-Silva, V. D., et al. (2010). Lista anotada de los anfibios y reptiles del estado de Hidalgo, México. México, D.F.: Universidad Autónoma del Estado de Hidalgo y Comisión Nacional para el Conocimiento y Uso de la Biodiversidad.

Roth-Monzón, A. (2010). Herpetofauna del municipio de Nopala de Villagrán, Hidalgo. Tesis. Facultad de Ciencias, Universidad Nacional Autónoma de México.

Roth-Monzón, A. J., Mendoza-Hernández, A. A. y Flores-Villela, O. A. (2011). Lampropeltis ruthveni (Serpentes: Colubridae) from the state of Hidalgo, Mexico. The Southwestern Naturalist, 56, 430-431.

Rzedowski, J. R. (2006). Vegetación de México. México, D.F.: Comisión Nacional para el Conocimiento y Uso de la Biodiversidad.

Semarnat (Secretaría de Medio Ambiente y Recursos Naturales). (2010). Norma Oficial Mexicana NOM-059-SEMARNAT-2010, Protección ambientalEspecies nativas de México de flora y fauna silvestres-Categorías de riesgo y especificaciones para su inclusión, exclusión o cambio-Lista de especies en riesgo. Diario Oficial de la Federación. 30 de diciembre de 2010, Segunda Sección. México.

Shine, R. (1995). A new hypothesis for the evolution of viviparity in reptiles. The American Naturalist, 145, 809-823.

Sperry, J. H., Ward, M. P. y Weatherhead, P. J. (2013). Effects of temperature, moon phase, and prey on nocturnal rattlesnakes: an automated telemetry study. Journal of Herpetology, 47, 105-111.
Stewart, J. R. y Blackburn, D. (2014). Viviparity and placentation in lizards. En J. L. Rheubert, D. S. Siegel y S. E. Trauth (Eds.), Reproductive biology and phylogeny of lizards and tuatara (pp. 448-563). Enfield, New Hampshire: CRC Press.

Suazo-Ortuño, I., Alvarado-Díaz, J. y Martínez-Ramos, M. (2008). Effects of conversion of dry tropical forest to agricultural mosaic on herpetofaunal assemblages. Conservation Biology, 22, 362-374.

Uetz, P. (2015). The reptile database. Recuperado el 1 febrero de 2015 de: http://reptile-database.org/.

Uribe-Peña, Z., Ramírez-Bautista, A. y Casas-Andreu, G. (1999). Anfibios y reptiles de las serranías del Distrito Federal. Cuadernos del Instituto de Biología No. 32. México, D.F.: Universidad Nacional Autónoma de México.

Vázquez-Díaz, J. y Quintero-Díaz, G. (2005). Anfibios y reptiles de Aguascalientes. México, D.F.: Comisión Nacional para el Conocimiento y Uso de la Biodiversidad.

Vite-Silva, V. D. (2008). Lista anotada de anfibios y reptiles en la Reserva de la Biosfera Barranca de Metztitlán, Hidalgo, México. Tesis. Universidad Autónoma del Estado de Hidalgo.

Vite-Silva, V. D., Ramírez-Bautista, A. y Hernández-Salinas, U. (2010). Diversidad de anfibios y reptiles de la Reserva de la Biosfera Barranca de Metztitlán, Hidalgo, México. Revista Mexicana de Biodiversidad, 81, 473-485.

Vitt, L. J. y Caldwell, J. P. (2014). Herpetology, an introductory biology of amphibians and reptiles. San Diego, California: Elsevier.

Wake, M. H. (1993). Evolution of oviductal gestation in anphibians. The Journal of Experimental Zoology, 266, 394-413.

Wilson, L. D., Mata-Silva, V. y Johnson, J. D. (2013a). A conservation reassessment of the amphibians of Mexico based on the EVS measure. Amphibian and Reptile Conservation, 7, 97-127.

Wilson, L. D., Mata-Silva, V. y Johnson, J. D. (2013b). A conservation reassessment of the reptiles of Mexico based on the EVS measure. Amphibian and Reptile Conservation, 7, 1-47. 